\title{
Optimal Combination of Aircraft Maintenance Tasks by a Novel Simplex Optimization Method
}

\author{
Huaiyuan Li, ${ }^{1}$ Hongfu Zuo, ${ }^{1}$ Dan Lei, ${ }^{2}$ Kun Liang, ${ }^{1,3}$ and Tingting Lu \\ ${ }^{1}$ College of Civil Aviation, Nanjing University of Aeronautics and Astronautics, Nanjing, Jiangsu 211106, China \\ ${ }^{2}$ Jiangshan Tourism Administration, Quzhou, Zhejiang 324100, China \\ ${ }^{3}$ Traffic Engineering Institute, Huaiyin Institute of Technology, Huai'an, Jiangsu 223003, China
}

Correspondence should be addressed to Huaiyuan Li; 995657041@qq.com

Received 9 January 2015; Accepted 2 June 2015

Academic Editor: George S. Dulikravich

Copyright (C) 2015 Huaiyuan Li et al. This is an open access article distributed under the Creative Commons Attribution License, which permits unrestricted use, distribution, and reproduction in any medium, provided the original work is properly cited.

\begin{abstract}
Combining maintenance tasks into work packages is not only necessary for arranging maintenance activities, but also critical for the reduction of maintenance cost. In order to optimize the combination of maintenance tasks by fuzzy C-means clustering algorithm, an improved fuzzy C-means clustering model is introduced in this paper. In order to reduce the dimension, variables representing clustering centers are eliminated in the improved cluster model. So the improved clustering model can be directly solved by the optimization method. To optimize the clustering model, a novel nonlinear simplex optimization method is also proposed in this paper. The novel method searches along all rays emitting from the center to each vertex, and those search directions are rightly $n+1$ positive basis. The algorithm has both theoretical convergence and good experimental effect. Taking the optimal combination of some maintenance tasks of a certain aircraft as an instance, the novel simplex optimization method and the clustering model both exhibit excellent performance.
\end{abstract}

\section{Introduction}

Combining maintenance tasks into work packages is not only necessary for arranging maintenance activities, but also critical for the reduction of maintenance cost. In the field of civil aircraft maintenance, in order to reduce the maintenance cost, the number of character check is constantly increased. For example, the number of $A$ check is increased from 64 to 128 . Recently, character checks have been completely cancelled, instead, the maintenance tasks executed in character check are combined into several small maintenance work packages and are equally allocated to line maintenance, so as to balance the maintenance and reduce the maintenance cost. However, it is still inevitable to optimally combine maintenance tasks into work packages no matter whether character check is utilized or not. As combinatorial optimizations usually belong to NP-hard problems that are difficult to be exactly solved, only optimal approximation solutions are obtained on the basis of fuzzy clustering of maintenance tasks in the paper. First of all, a modification is made on the fuzzy $\mathrm{C}$-means clustering model to reduce the dimension. Then, a novel nonlinear simplex algorithm is put forward. Lastly, the optimal combination of maintenance tasks is transformed into a clustering problem which can be solved by a novel simplex algorithm.

In the traditional fuzzy C-means (FCM) clustering method, not only the degree of fuzzy membership is taken as an independent variable but also the center of every class is unknown. For this reason, the FCM model is a high-dimension optimization problem that is difficult to be directly solved. Usually, only its local optimal solution can be found out by the alternative optimization algorithm. Therefore, the traditional FCM may present high error rate and poor adaptability. In this paper, class centers are regarded as a function of fuzzy membership degree and samples; thus, an improved FCM model is developed. The improved model does not explicitly include clustering centers, so the number of unknown variables is reduced, which not only results in 
the decrease in the computational cost but also makes it possible to directly solve the clustering model by the optimization method. According to the experimental results, if we directly solve the improved FCM model, the results of the improved FCM model are better than those of traditional FCM model in most cases.

As a direct optimization method, the traditional nonlinear simplex method mainly has the following serious defects: poor efficiency in solving high dimension problems and rather slow calculating speed [1]. In high-dimension problems, a sufficient number of vertexes are needed to form a simplex, and the descending directions are more complex. However, the traditional simplex optimization method only searches in a single one direction and merely updates one vertex at each iteration, when it optimizes by reflection, expansion and contraction [2]. Even though the new vertex is much better than the former one, only a small number of vertexes are updated. Thus, the number of vertexes updated at each iteration is too small, which is not enough for the rapid revolution of the entire simplex. Namely, update of one vertex hardly contributes to the evolution of high-dimension simplex comprised with a large amount of vertexes. Hence, in the paper, center ray operation is introduced, which can search along all rays emitting from the center to each vertex. By the center ray operation, more search directions are available and most of vertexes in the simplex can be updated, which will greatly accelerate the optimization speed and improve capacity of solving high-dimension problems. Experiments show that the slow computing speed of the traditional simplex method is mainly caused by several reasons: (1) too many shrinking operations consume great computation cost [3]; (2) fixed search speed and step can not adapt to different conditions [4]; (3) the search direction is not necessarily accurate descent direction, which results in lower solving efficiencies [5]. In view of the above mentioned reasons, shrinkage operation is removed in the paper. In case of slow evolution of simplex, the novel simplex method regenerates a new small simplex according to the current estimated descent direction. This not only accelerates the optimization speed and reduces computational expense but also avoids the degeneration problem that easily occurs in the evolution course of traditional simplex method.

The paper consists of three aspects. Firstly, efficiency of the traditional simplex method is low, especially when it is utilized to optimize high-dimension problems [6]. Accordingly, in the paper, a novel nonlinear simplex optimization method is primarily put forward, which can not only search along all rays emitting from the center to each vertex but also simultaneously update numerous vertexes. Secondly, concerning the problems of too many variables and large calculated amount in the traditional FCM, the FCM model is improved in the paper. Finally, optimal combination of maintenance tasks is concluded as a problem of constrained clustering, the novel simplex method is employed to solve the improved FCM model so as to achieve the optimal combination of maintenance tasks.

\section{Improvement of Fuzzy C-Means Clustering}

2.1. Introduction to Traditional FCM. Clustering means adaptively classifying the sample set $\left\{t_{j}, j=0, \ldots, n\right\}$ into $c$ classes according to the similarity among samples. One of the most common methods of clustering is fuzzy C-means clustering [7]. If $u_{i j} \in[0,1]$ indicates fuzzy membership that the sample $t_{j}$ affiliates the $i$ th class and $c_{i}$ is the center of the sample of the $i$ th class, FCM takes $d_{i j}^{2}=\left\|t_{j}-c_{i}\right\|^{2}$ as quantitative index to measure the difference or dissimilarity between $t_{j}$ and $c_{i}$. Assuming the fuzzy control parameter $m>1$, the FCM model is as

$$
\begin{array}{ll}
\min & J_{m}=\sum_{i=1}^{c} \sum_{j=1}^{n} u_{i j}^{m} d_{i j}^{2} \\
\text { s.t. } & \sum_{i=1}^{c} u_{i j}=1, \quad \forall j=1, \ldots, n \\
& u_{i j} \geq 0, \quad \forall i=1, \ldots, c, \quad j=1, \ldots, n .
\end{array}
$$

The key to the traditional FCM algorithm is to solve the class center matrix $\left[c_{i}\right]_{c \times d}$ and the fuzzy membership matrix $\left[u_{i j}\right]_{c \times n}$. However, clustering is a NP problem, and (1) is usually a high-dimension optimization problem with numerous local minimum values [8]. Traditionally, Model (1) is alternatively solved by the steepest descent method. Firstly, assuming that the current clustering center $c_{i}$ is known, and the stationary point of Model (1) with respect to $u_{i j}$ is taken as the next $u_{i j}$. Then, the new $u_{i j}$ is supposed to be constant, and the optimum $c_{i}$ is achieved with the stationary point of Model (1) with respect to $c_{i}$, as shown in

$$
c_{i}=\sum_{j=1}^{n} \frac{u_{i j}^{m}}{\sum_{k=1}^{n} u_{i k}^{m}} x_{j} .
$$

In the whole course of computation, the unknown $c_{i}, u_{i j}$, $i=1, \ldots, c, j=1, \ldots, n$ are not simultaneously computed as a whole, and also, the correlation between $c_{i}$ and $u_{i j}$ is ignored. Therefore, only the local minimum point can be found out. The traditional FCM model must repeatedly compute the distance between each sample and all centers of class at each iteration, which consumes a large amount of computation.

2.2. Improved Model of FCM. Actually, there is a close correlation between $c_{i}$ and $u_{i j}$. According to (2), if the class center $c_{i}$ is regarded as the function of $u_{i j}$ and $x_{j}$, and then $c_{i}$ can be eliminated and the number of variables will be reduced. From the property of norm and inner product, $d_{i j}^{2}$ can be denoted as $d_{i j}^{2}=\left\langle t_{j}, t_{j}\right\rangle-2 \sum_{l=1}^{n} u_{i l}^{m}\left\langle t_{j}, t_{l}\right\rangle / \sum_{k=1}^{n} u_{i k}^{m}+$ $\sum_{p=1}^{n} \sum_{q=1}^{n} u_{i p}^{m} u_{i q}^{m}\left\langle t_{p}, t_{q}\right\rangle /\left(\sum_{k=1}^{n} u_{i k}^{m}\right)^{2}$. As such, in the paper, (2) is substituted into Model (1) directly and the FCM model is obtained as (3), whose variables only contain $u_{i j}$ not $c_{i}$ : 


$$
\begin{aligned}
\min & J_{m}=\sum_{i=1}^{c} \sum_{j=1}^{n} u_{i j}^{m} d_{i j}^{2}=\sum_{i=1}^{c} \sum_{j=1}^{n} u_{i j}^{m}\left(\left\langle t_{j}, t_{j}\right\rangle-\frac{2 \sum_{l=1}^{n} u_{i l}^{m}\left\langle t_{j}, t_{l}\right\rangle}{\sum_{k=1}^{n} u_{i k}^{m}}+\frac{\sum_{p=1}^{n} \sum_{q=1}^{n} u_{i p}^{m} u_{i q}^{m}\left\langle t_{p}, t_{q}\right\rangle}{\left(\sum_{k=1}^{n} u_{i k}^{m}\right)^{2}}\right) \\
\text { s.t. } & \sum_{i=1}^{c} u_{i j}=1, \quad \forall j=1, \ldots, n \\
& u_{i j} \geq 0, \quad \forall i=1, \ldots, c, j=1, \ldots, n .
\end{aligned}
$$

Different from (1), (3) does not contain class center $c_{i}$. Instead, it is composed of inner products among samples. If they can be computed and stored in advance, the computed amount of Model (3) can be reduced especially when sample size is large. Compared with (1), (3) contains much less unknown variables, which makes it possible to directly solve (3). As (3) has numerical stationary points with respect to $u_{i j}$, the optimization method which needs derivative is inappropriate for (3). Inspired by the traditional simplex algorithm, a novel direct optimization method that can be utilized to solve nonlinear programming, namely, a novel simplex optimization method based on center ray, is presented to solve (3) in the paper.

2.3. The Verification of Improved Model of FCM. To solve the constrained optimization Model (3), (3) is firstly transformed into the unconstrained Model (4) by the exterior penalty function method. Then, the novel optimization method proposed in the paper is employed to solve the unconstrained problem, namely, (4):

$$
\begin{aligned}
& \min J_{m}=\sum_{i=1}^{c} \sum_{j=1}^{n} u_{i j}^{m}\left(\left\langle t_{j}, t_{j}\right\rangle-\frac{2 \sum_{l=1}^{n} u_{i l}^{m}\left\langle t_{j}, t_{l}\right\rangle}{\sum_{k=1}^{n} u_{i k}^{m}}\right. \\
& \left.+\frac{\sum_{p=1}^{n} \sum_{q=1}^{n} u_{i p}^{m} u_{i q}^{m}\left\langle t_{p}, t_{q}\right\rangle}{\left(\sum_{k=1}^{n} u_{i k}^{m}\right)^{2}}\right) \\
& +\delta_{k}\left(\sum_{i=1}^{c} \sum_{j=1}^{n} \max ^{2}\left(0,-u_{i j}\right)+\sum_{j=1}^{n}\left(\sum_{i=1}^{c} u_{i j}-1\right)\right) .
\end{aligned}
$$

If $c_{i}$ is the center of the $i$ th class of simulation sample and $r_{l}$ is the random vector obeying certain distribution, then $C_{i}=$ $\left\{c_{i}+r_{l}, l=1, \ldots,\left|C_{i}\right|\right\}$ can be regarded as the set of the $i$ th class simulation sample. In the experiment, $r_{l}$, respectively, follows normal distribution and uniform distribution, in other words, simulate, respectively, under $r_{l} \sim N\left(0,\left(\left(c_{i+1}-c_{i}\right) / 2\right)^{2}\right)$ and $r_{l} \sim U\left(-\left(\left(c_{i+1}-c_{i}\right) / 2\right),\left(c_{i+1}-c_{i}\right) / 2\right)$ circumstance. Three classes of eight-dimensional random vectors are randomly generated, which comprise sample set $\left\{C_{1}, C_{2}, C_{3}\right\}$, where, respectively, set $c_{1}=1, c_{2}=2$, and $c_{3}=3$. Before the sample is generated, the number of every class sample is randomly determined by $\left|C_{i}\right|=\left\lfloor\kappa_{i}+\vartheta\left(r_{i}-0.5\right)\right\rfloor$, where, $\vartheta=5$, $r_{i} \sim U(0,1)$ and $\kappa_{1}=15, \kappa_{2}=20, \kappa_{3}=25$.
Generally, the index $V_{p e}=-(1 / n) \sum_{i=1}^{c} \sum_{j=1}^{n} u_{i j} \log u_{i j}$, $V_{m p c}=1-(c /(c-1))\left(1-(1 / n) \sum_{i=1}^{c} \sum_{j=1}^{n} u_{i j}^{2}\right)$, $V_{x b}=\sum_{i=1}^{c} \sum_{j=1}^{n} u_{i j}^{m}\left\|t_{j}-c_{i}\right\|^{2} /\left(n \min _{i \neq j}\left\|c_{i}-c_{j}\right\|^{2}\right), V_{k}=$ $\left(\sum_{i=1}^{c} \sum_{j=1}^{n} u_{i j}^{m}\left\|t_{j}-c_{i}\right\|^{2}+(1 / c) \sum_{i=1}^{c}\left\|c_{i}-\bar{c}\right\|^{2}\right) / \min _{i \neq j}\left\|c_{i}-c_{j}\right\|^{2}$, and the error rate of classification is utilized to evaluate the effect of the classification and compare the performance of the clustering model. Model (4) is solved by Algorithm 5. In the experiment, numerical simulation is repeated 300 times under the condition of same experimental parameters and some average results are totaled, which are tabulated in Table 1. $J_{m}$ in Table 1 is the practical optimum value of the clustering object function, which can indicate whether the clustering model has advantages and disadvantages. In the experiment, the main parameters of Algorithm 5 are as follows: $\dddot{\lambda}_{h}=0.985, t_{\max }=10, \rho_{\text {nei }}=0.1, h_{\text {small }}=0.1, h_{0}=0.5$, $\alpha_{\text {init }}^{\text {forward }}=2.2, \alpha_{\text {end }}^{\text {forward }}=0.75, \alpha_{\text {init }}^{\text {reverse }}=-2.2, \alpha_{\text {end }}^{\text {reverse }}=-1.2$, $\mu_{\text {inner }}=0.5, \mu_{\text {outer }}=1.3, \alpha^{f e}=\alpha^{r e}=\mu^{e}=1.35$, $\alpha^{f c}=\mu^{o c}=0.7, \mu^{i c}=1.5, \alpha^{s}=\mu^{s}=-0.5, \dot{\lambda}_{h}=3, \ddot{\lambda}_{h}=0.95$, $\alpha^{i c}=0.5, \alpha^{f_{s}}=-0.4, \alpha^{r s}=0.4$, and $\mu^{s}=0.35$.

After analyzing the results in Table 1, the following conclusion is obtained. $J_{m}$ in Model (3) is always smaller than that in Model (1), which implies the result of Model (3) is better than that of Model (1) from the view of mathematical optimization of object function. $V_{x b}, V_{k}$ of Model (3) are also better than those of Model (1), which shows that, after clustering of Model (3), the interior of each class becomes more compact and the separability and dissimilarity between classes are more significant apparent. Besides, in terms of the classification error rate, Model (3) is also more accurate than Model (1). However, if $V_{p e}$ and $V_{m p c}$ are taken into consideration, Model (1) seems to be better, indicating that makingdecisions of Model (1) are clearer and more arbitrary while the membership degree of Model (3) is more uniform than that of Model (1). Thus, compared with traditional FCM, Model (3) is stronger in adaptability and higher in recognition accuracy.

\section{A Novel Simplex Optimization Algorithm}

3.1. Description of the Novel Simplex Method. The convex hull, namely, the hyper polyhedron composed of $n+1$ vertexes in $R^{n}$ is called a simplex. Assuming vertexes $x_{0}, x_{1}, \ldots, x_{n}$ in the simplex are sorted in ascending order by function values, the simplex of the $k$ th iteration is denoted as $S_{k}=\left\{x_{0}, \ldots, x_{n}\right\}$. Obviously, $n$ side vectors $x_{i}-x_{j}, j=0, \ldots, n \wedge j \neq i$, connecting with the $i$ th vertex $x_{i}$ are linearly independent, 
TABLE 1: Comparison of clustering results.

\begin{tabular}{|c|c|c|c|c|c|c|c|}
\hline Distribution & Model & $V_{p e}$ & $V_{m p c}$ & $V_{x b}$ & $V_{k}$ & Error rate & $J_{m}$ \\
\hline Uniform & Model (1) & 0.050742 & 0.974330 & 0.079669 & 0.095152 & 0.007974 & 27.780529 \\
\hline Uniform & Model (3) & 0.372381 & 0.729550 & 0.073406 & 0.088955 & 0.000000 & 25.252153 \\
\hline Norm & Model (1) & 0.224796 & 0.836828 & 0.209994 & 0.225994 & 0.005346 & 72.118062 \\
\hline Norm & Model (3) & 0.638111 & 0.467340 & 0.203394 & 0.220157 & 0.004615 & 61.697758 \\
\hline
\end{tabular}

from which the degeneration of the simplex can be judged. The whole iteration of the traditional nonlinear simplex algorithm mainly consists of four steps: reflection, expansion, contraction, and shrinkage. In contrast, the novel algorithm in the paper primarily solves problems by generating a simplex, raying outward from the simplex center and reflecting operations. Taking the unconstrained optimization problem min $f(x)$ as an example, the novel simplex algorithm can be described as below.

3.1.1. Generation of a Simplex. Due to the tendency of deformation and degeneration in the process of the simplex's iteration, it is usually better that the initial simplex is constructed as an orthogonal one. When one side of the orthogonal simplex gets close to the negative gradient direction $-\rho_{k}$, the simplex will search much rapidly. If $A_{i}=\left(\tau_{1}, \ldots, \tau_{i}\right)$ and $z$ is any of solution of the linear equations $A_{i}{ }^{T} Z=0$, then $z$ will be orthogonal to $\tau_{1}, \ldots, \tau_{i}$. Firstly, we set $\tau_{1}=-\rho_{k}$ and $A_{1}=\left(\tau_{1}\right)$. If $z$, that is, any of the solutions of $A_{i}{ }^{T} Z=0$, is taken as the last column of $A_{i}$ to generate a new matrix $A_{i+1}$, then the orthogonal matrix $A_{n}=\left(\tau_{1}, \ldots, \tau_{n}\right)$ can be obtained after repeatedly solving the linear equations $n-1$ times, where, $\tau_{1}, \ldots, \tau_{n}$ represent $n$ orthogonal vectors. Subsequently, we choose the point whose function value is better from $\left\{z_{k}+\right.$ $\left.h_{m} \tau_{i}, z_{k}-h_{m} \tau_{i}\right\}$ as the vertex of the simplex. In this paper, the flow of simplex generation is shown as Algorithm 1 .

\section{Algorithm 1.}

Step 1. Let $\tau_{1}=-\rho_{k} /\left\|\rho_{k}\right\|, i=1, S_{k}=\left\{z_{k}\right\}, A_{1}=\left[\tau_{1}\right]$.

Step 2. Let

$$
\begin{aligned}
& S_{k} \\
& \quad= \begin{cases}S_{k} \cup\left\{z_{k}+h_{m} \tau_{i}\right\} & f\left(z_{k}+h_{m} \tau_{i}\right)<f\left(z_{k}-h_{m} \tau_{i}\right) \\
S_{k} \cup\left\{z_{k}-h_{m} \tau_{i}\right\} & \text { others. }\end{cases}
\end{aligned}
$$

Step 3. Solve the linear equations $A_{i}{ }^{T} Z=0$ and choose any of the solutions as $z$ and let

$$
\begin{aligned}
\tau_{i+1} & =\frac{z}{\|z\|}, \\
A_{i+1} & =\left[\tau_{1}, \ldots, \tau_{i}, \tau_{i+1}\right] .
\end{aligned}
$$

Step 4. When $i<n-1$, then $i=i+1$ and go to Step 2; otherwise exit.
Following the procedure, the simplex $S_{k}$ is generated at $z_{k}$. Edges connecting with $z_{k}$ are orthogonal vectors whose lengths are all $h_{m}$. Besides, the first edge is parallel to the negative gradient direction $-\rho_{k}$.

\subsubsection{Center Ray Operation}

Theorem 2. If the center of the simplex is denoted as $\bar{x}=$ $(1 /(n+1)) \sum_{i=0}^{n} x_{i}$ and the direction from the center to the ith vertex is denoted as $u_{i}=x_{i}-\bar{x}, i=0, \ldots, n$, then $U=\left\{u_{i} \mid i=\right.$ $0, \ldots, n\}$ is a group of positive base.

Proof. Obviously, $u_{i}=x_{i}-\bar{x}=x_{i}-(1 /(n+1)) \sum_{j=0}^{n} x_{j}=$ $-(1 /(n+1)) \sum_{j=0, j \neq i}^{n}\left(x_{j}-x_{i}\right)$. Assuming there exist constants $l_{1}, \ldots, l_{i}, \ldots, l_{n}$ who are not all zero such that $\sum_{i=1}^{n} l_{i} u_{i}=0$ is tenable, then $\sum_{i=1}^{n} l_{i} u_{i}=(-1 /(n+1)) \sum_{i=1}^{n}\left(l_{i} \sum_{j=0, j \neq i}^{n}\left(x_{j}-x_{i}\right)\right)=$ $(-1 /(n+1)) \sum_{i=1}^{n}\left(n l_{i}-\sum_{j=1, j \neq i}^{n} l_{j}\right)\left(x_{0}-x_{i}\right)=0$. As the side vectors $x_{0}-x_{i}, i=1, \ldots, n$, are linearly independent, we get $n l_{i}-\sum_{j=1, j \neq i}^{n} l_{j}=0, i=1, \ldots, n$, from which $l_{i}=0, i=1, \ldots, n$ is obtained. Therefore, the assumption is contradictory and untenable. Thus, $u_{i}, i=1, \ldots, n$ is linearly independent, and $\operatorname{rank} U=n$ is obtained. Therefore, $U$ is a spanning set of $R^{n}$. On the other hand, $\sum_{i=0}^{n} u_{i}=\sum_{i=0}^{n}\left(x_{i}-\bar{x}\right)=\sum_{i=0}^{n} x_{i}-(n+1) \bar{x}=$ 0 . In addition, the number of vectors is exactly right $n+1$. According to literatures [9-11], $u_{i}, i=0, \ldots, n$ are positive bases.

The center ray operation searches along the direction of $u_{i}, i=0, \ldots, n$ by comparing $f(\bar{x})$ and $f\left(x_{i}\right), i=0, \ldots, n$. Firstly, as shown in (7), the first search is along the direction $u_{i}, i=0, \ldots, n$, where, $\mu_{i}$ must satisfy (8), and it is advised to calculate $\mu_{i}$ by (9) or (10). To be specific, when $f(\bar{x})>f\left(x_{i}\right)$, we let $\mu_{i}=\mu_{\text {outer }}>1$, which means search outside the simplex. If $f(\bar{x}) \leq f\left(x_{i}\right)$, it is reasonable we assume better points locate between $\bar{x}$ and $x_{i}$, so we let $\mu_{i}=\mu_{\text {inner }} \in(0,1)$ in order to search inside the simplex:

$$
\begin{aligned}
\widehat{x}_{i}^{e} & =\bar{x}+\mu_{i}\left(x_{i}-\bar{x}\right), \quad i=0, \ldots, n, \\
\mu_{i} & = \begin{cases}\mu_{\text {inner }} \in(0,1) & f(\bar{x}) \leq f\left(x_{i}\right) \\
\mu_{\text {outer }} \in(1, \infty) & \text { others, }\end{cases} \\
\mu_{\text {outer }} & =\dot{\mu}_{\text {outer }}, \\
\mu_{\text {inner }} & =\dot{\mu}_{\text {inner }},
\end{aligned}
$$




$$
\begin{aligned}
& \mu_{\text {outer }}=\mu_{\text {outer }}^{\text {init }}+\frac{\mu_{\text {outer }}^{\text {end }}-\mu_{\text {outer }}^{\text {init }}}{t_{\max }} t, \\
& \mu_{\text {inner }}=\mu_{\text {inner }}^{\text {init }}+\frac{\mu_{\text {inner }}^{\text {end }}-\mu_{\text {inner }}^{\text {init }}}{t_{\text {max }}} t .
\end{aligned}
$$

Next, in order to look for a better point, the second detection is attempted according to $f\left(\widehat{x}_{i}^{e}\right)$, indicated in (11). The second detection point is located by parameter $\mu^{q}$ whose scope is determined by comparing function values of $\hat{x}_{i}^{e}, x_{i}, \bar{x}$, as indicated in (12):

$$
\begin{aligned}
& \tilde{x}_{i}^{e}=\bar{x}+\mu^{q} \mu_{i}\left(x_{i}-\bar{x}\right), \quad i=0, \ldots, n, \\
& \mu^{q}= \begin{cases}\mu^{e} \in(1, \infty) & f\left(\hat{x}_{i}^{e}\right)<f\left(x_{i}\right)<f(\bar{x}) \\
\mu^{o c} \in\left(\mu_{i}^{-1}, 1\right) & f\left(x_{i}\right) \leq f\left(\hat{x}_{i}^{e}\right) \leq f(\bar{x}) \\
\mu^{i c} \in\left(1, \mu_{i}^{-1}\right) & f(\bar{x}) \leq f\left(x_{i}\right) \\
\mu^{s} \in\left(0, \mu_{i}^{-1}\right) & f\left(x_{i}\right)<f(\bar{x}) \leq f\left(\hat{x}_{i}^{e}\right) .\end{cases}
\end{aligned}
$$

If $\widehat{x}_{i}^{e}$, which is found out by the first search, is the minimum point, then the direction is supposed as the descent direction. Naturally, a better point may be acquired after extending forward along the same direction. So let $\mu^{q}>1$ in the case. If expansion point $\widetilde{x}_{i}^{e}$ is better than $\widehat{x}_{i}^{e}, \widetilde{x}_{i}^{e}$ will be accepted; or else, $\hat{x}_{i}^{e}$ will be accepted. When $f\left(x_{i}\right) \leq f\left(\hat{x}_{e}\right) \leq$ $f(\bar{x}) \vee f(\bar{x})<f\left(x_{i}\right)$, better points are supposed to locate between $x_{i}$ and $\widehat{x}_{e}$. Thus, set $\mu_{i}^{-1}<\mu^{q}<1$, and the algorithm goes back for a certain distance to look for a better point $\tilde{x}_{i}^{e}$. Then, $x_{i}$ is replaced with the better point from $\tilde{x}_{i}^{e}$ and $\hat{x}_{i}^{e}$, as a result, a new simplex will be formed. When $f\left(x_{i}\right)<f(\bar{x}) \leq$ $f\left(\widehat{x}_{e}\right)$, assumption that better points locate between $x_{i}$ and $\bar{x}$ is reasonable; thus, let $\mu_{i}^{-1}<\mu^{q}<0$. If $\tilde{x}_{i}^{e}$ is better than $x_{i}, x_{i}$ is replaced with $\tilde{x}_{i}^{e}$. Otherwise, vertex $x_{i}$ still retains in the new simplex. Through the comparison between $f\left(x_{i}\right)$ and $f(\bar{x})$, center ray operation can search both inside and outside the simplex, so the algorithm can get close to the extreme point no matter it is inside or outside the simplex. Some illustrations of the center ray operation under 2-dimensional circumstance are shown in Figure 1.

In the process of the center ray, the simplex can become larger or smaller. The adaptive change can reduce calculated amount and accelerate the convergence. If search direction is appropriate, the second search can expand outwards, which can enlarge the simplex and speed up the search. If the extreme point is inside the simplex, the simplex can be automatically downsized by the comparison of function values among the center, the vertex and the detected point. Consequently, the convergence will be accelerated. The search of traditional simplex method relies mainly on reflection operations; unfortunately, its search is only along one direction and only one vertex is updated. Computation of the traditional method is slow and its effect is poor in solving high-dimension problems. In comparison, the center ray operation searches along directions of $n+1$ positive bases, and one operation may update several vertexes. As a result, not only the computation speed is improved but also the omission of solution is avoided.

3.1.3. Reflection Operation. In the simplex, the center of all vertexes except the worst vertex $x_{n}$ is denoted as $\bar{x}^{\prime}$, as shown (13). Firstly, like the traditional simplex method, $x_{n}$ is reflected about $\bar{x}^{\prime}$. As indicated in (14), $\widehat{x}^{r}$, namely, the reflection point of $x_{n}$ about $\bar{x}^{\prime}$, is computed. Different from the traditional simplex method, the algorithm in the paper can search along two inverse reflection directions by comparison between $f\left(x_{n}\right)$ and $f\left(\bar{x}^{\prime}\right)$, as shown in (15). Besides, like center ray operation, there are two attempts in reflection operation, as shown in (14) and (18), respectively:

$$
\begin{aligned}
\bar{x}^{\prime} & =\sum_{i=0}^{n-1} x_{i}, \\
\widehat{x}^{r} & =\bar{x}^{\prime}+\alpha\left(\bar{x}^{\prime}-x_{n}\right), \\
\alpha & = \begin{cases}a^{\text {forward }}>0 & f\left(x_{n}\right) \geq f\left(\bar{x}^{\prime}\right) \\
a^{\text {reverse }}<-1 & f\left(x_{n}\right)<f\left(\bar{x}^{\prime}\right) .\end{cases}
\end{aligned}
$$

The reflection coefficient $\alpha$ can be computed by either (16) or (17). As shown in (16), the reflection coefficient $\alpha$ is usually constant. When $\alpha$ decreases with iterations as shown in (17), the computation speed can be accelerated. In (17), $t_{\max }$ indicates the preset maximum iterations, $\alpha_{\text {init }}^{\text {forward }}, \alpha_{\text {init }}^{\text {reverse }}$ are the reflection coefficients when $t=0$; and $\alpha_{\text {end }}^{\text {forward }}$ and $\alpha_{\text {end }}^{\text {reverse }}$ are the reflection coefficients when $t=t_{\max }$ :

$$
\begin{aligned}
\alpha^{\text {forward }} & =\dot{\alpha}^{\text {forward }}, \\
\alpha^{\text {reverse }} & =\dot{\alpha}^{\text {reverse }}, \\
\alpha^{\text {forward }} & =\alpha_{\text {init }}^{\text {forward }}-\frac{\alpha_{\text {init }}^{\text {forward }}-\alpha_{\text {end }}^{\text {forward }}}{t_{\max }} t ; \\
\alpha^{\text {reverse }} & =\alpha_{\text {init }}^{\text {reverse }}-\frac{\alpha_{\text {init }}^{\text {reverse }}-\alpha_{\text {end }}^{\text {reverse }}}{t_{\text {max }}} t ; \\
& 0 \leq \alpha_{\text {init }}^{\text {forward }} \leq \alpha_{\text {init }}^{\text {forward }},
\end{aligned}
$$

After that, by comparison among $f\left(\widehat{x}^{r}\right), f\left(\bar{x}^{\prime}\right), f\left(x_{n}\right), \widetilde{x}^{r}$ is computed by (18) to the second search. When $f\left(x_{n}\right) \geq$ $f\left(\bar{x}^{\prime}\right)$, coefficient $\alpha_{q}$ must satisfy (19); when $f\left(x_{n}\right)<f\left(\bar{x}^{\prime}\right)$, $\alpha_{q}$ must satisfy (20). The final reflection point is denoted as $x^{r}$, as shown in (21). If $f\left(x^{r}\right)<f\left(x_{n}\right), x_{n}$ is replaced with $x^{r}$ to form a new simplex. Otherwise, the reflection will fail. 

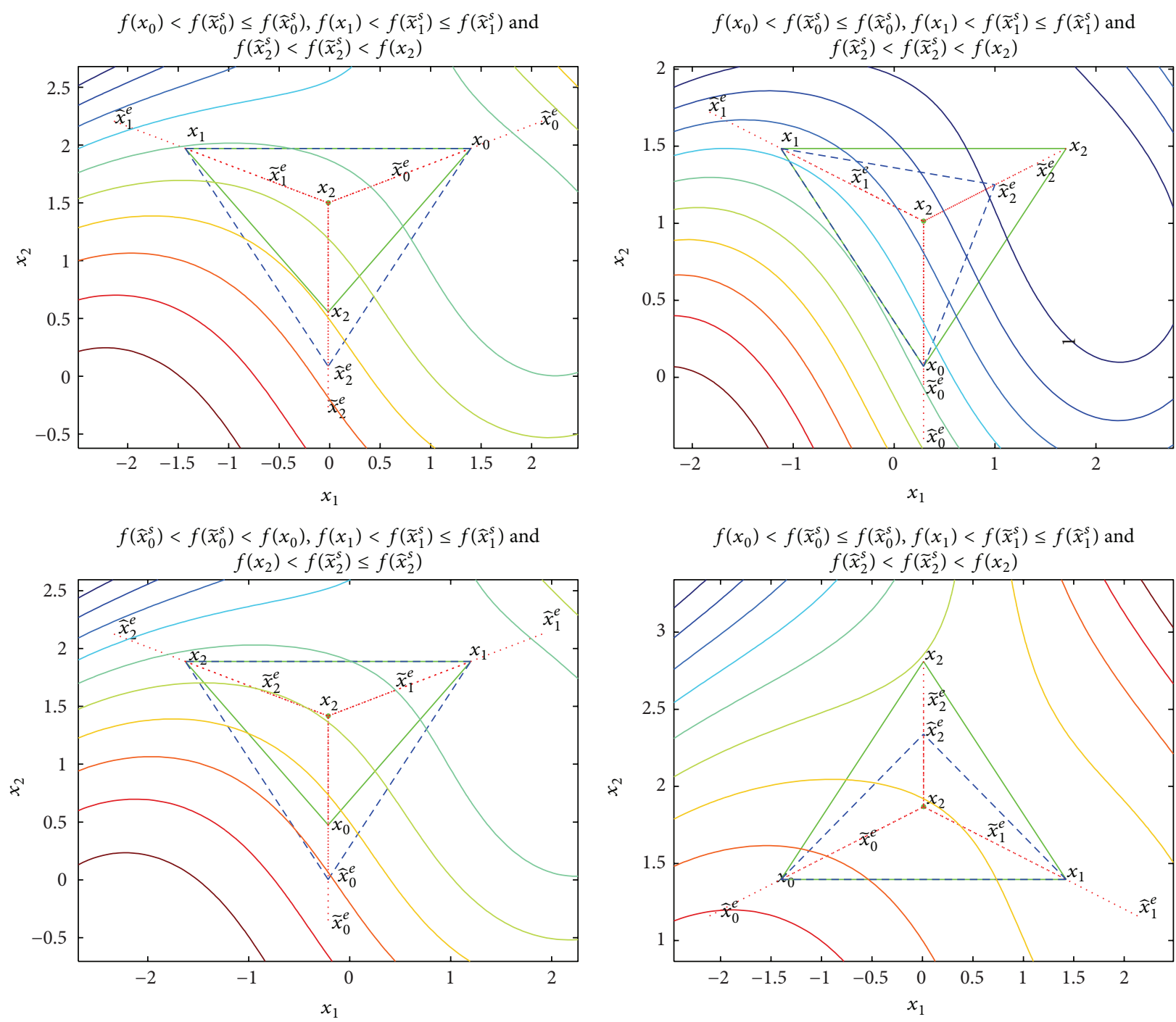

FIGURE 1: Illustration of center ray under 2-dimensional circumstance.

Figure 2 illustrates some of the process of reflection operations under 2-dimensional circumstance:

$$
\begin{aligned}
& \tilde{x}^{r}=\bar{x}^{\prime}+\alpha_{q} \alpha\left(\bar{x}^{\prime}-x_{n}\right), \\
& \alpha_{q}= \begin{cases}\alpha^{f e} \in(1, \infty) & f\left(\hat{x}^{r}\right)<f\left(\bar{x}^{\prime}\right) \leq f\left(x_{n}\right) \\
\alpha^{f c} \in(0,1) & f\left(\bar{x}^{\prime}\right) \leq f\left(\hat{x}^{r}\right)<f\left(x_{n}\right) \\
\alpha^{f s} \in\left(-\alpha^{-1}, 0\right) & f\left(\bar{x}^{\prime}\right) \leq f\left(x_{n}\right)<f\left(\hat{x}^{r}\right),\end{cases} \\
& \alpha_{q}= \begin{cases}\alpha^{r e} \in(1, \infty) & f\left(\hat{x}^{r}\right)<f\left(x_{n}\right)<f\left(\bar{x}^{\prime}\right) \\
\alpha^{r c} \in\left(-\alpha^{-1}, 1\right) & f\left(x_{n}\right) \leq f\left(\hat{x}^{r}\right)<f\left(\bar{x}^{\prime}\right) \\
\alpha^{r s} \in\left(0,-\alpha^{-1}\right) & f\left(x_{n}\right)<f\left(\bar{x}^{\prime}\right) \leq f\left(\hat{x}^{r}\right),\end{cases} \\
& x^{r}=\left\{\begin{array}{lll}
\hat{x}^{r} & f\left(\hat{x}^{r}\right)<f\left(\tilde{x}^{r}\right) \\
\tilde{x}^{r} & \text { others. }
\end{array}\right.
\end{aligned}
$$

Other than the traditional simplex method, both the center ray and the reflection in the paper twice searches along a same direction, likewise, object function is also computed twice. If results of the first detection are better, the algorithm outward expands along the same direction with a longer step to start the second search. Thus, computation speed will be accelerated. Otherwise, the algorithm will try to make certain contraction on the basis of the first search or search along inverse direction. This will improve the accuracy, and what is more important, this also can cause adaptive changes in both the size and the shape of the simplex in the search process. It means to automatically and continuously change the direction and step of the search, which enhance adaptability of the algorithm. One operation in the improved algorithm can achieve effects of several operations of the traditional algorithm, including reflection, expansion, contraction, and 

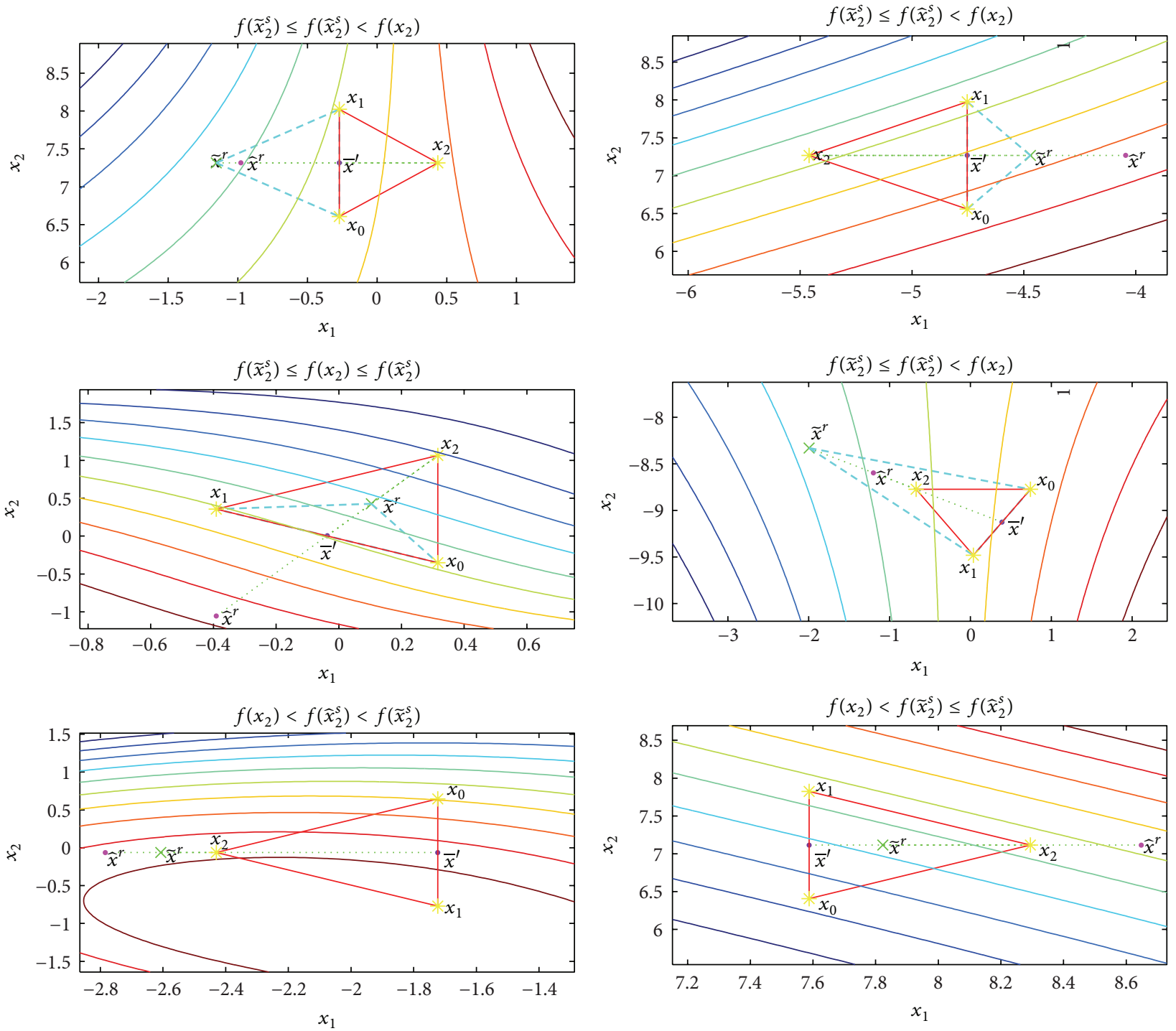

Figure 2: Illustration of the reflection operation under 2-dimensional circumstance.

shrinkage. As long as the appropriate parameters are chosen, the computation efficiency can be greatly improved.

3.1.4. Estimation of Gradient with Simplex. As derivative information is not utilized in the traditional simplex algorithm, the search is slow. Function values of at least $n+1$ points are known in the simplex of $n$-dimensional problems. Therefore, the gradient even the Hessian matrix at each iteration can be estimated by these $n+1$ points. When one side of the simplex is in accordance with the negative gradient, the simplex may search along the descent direction.

If the $i$ th side vectors $x_{i}-x_{0}(i=1, \ldots, n)$ connecting with the optimum point $x_{0}$ is denoted as $v_{i}$, then $v_{i}=\left\|v_{i}\right\| \vec{v}_{i}$, where $\left\|v_{i}\right\|=\left\|x_{i}-x_{0}\right\|$ is its side length and $\vec{v}_{i}$ is its direction of unit vector, as shown in (22). Since the directional derivative of $f(x)$ in the direction of $\vec{v}_{i}$ at $x_{0}$ is $\left.\left(\partial f(x) / \partial \vec{v}_{i}\right)\right|_{x=x_{0}}=$ $\lim _{t \rightarrow 0^{+}}\left(\left(f\left(x_{0}+t \vec{v}_{i}\right)-f\left(x_{0}\right)\right) / t\right), d_{i}$ in (23) is approximate to $\left.\left(\partial f(x) / \partial \vec{v}_{i}\right)\right|_{x=x_{0}}$ when the simplex is relatively small:

$$
\begin{aligned}
\vec{v}_{i} & =\frac{x_{i}-x_{0}}{\left\|x_{i}-x_{0}\right\|}, \\
d_{i} & =\left.\frac{f\left(x_{i}\right)-f\left(x_{0}\right)}{\left\|x_{i}-x_{0}\right\|} \approx \frac{\partial f(x)}{\partial \vec{v}_{i}}\right|_{x=x_{0}} .
\end{aligned}
$$

Suppose the gradient of $f(x)$ at $x_{0}$ is $\nabla f\left(x_{0}\right)$; then, $\vec{v}_{i}^{T} \nabla f\left(x_{0}\right)=\left.\left(\partial f(x) / \partial \vec{v}_{i}\right)\right|_{x=x_{0}} \approx d_{i}$. Let the matrix $V_{k}=$ $\left[\vec{v}_{1}, \vec{v}_{2}, \ldots, \vec{v}_{n}\right]^{T}$ and the vector $D_{k}=\left[d_{1}, d_{2}, \ldots, d_{n}\right]^{T}$; then, $V_{k} \nabla f\left(x_{0}\right) \approx D_{k}$, especially, when the simplex is relatively small. As $\vec{v}_{1}, \vec{v}_{2}, \ldots, \vec{v}_{n}$ are linearly independent, (24) can be regarded as the estimated value of the gradient:

$$
\rho_{k}=V_{k}^{-1} D_{k}
$$




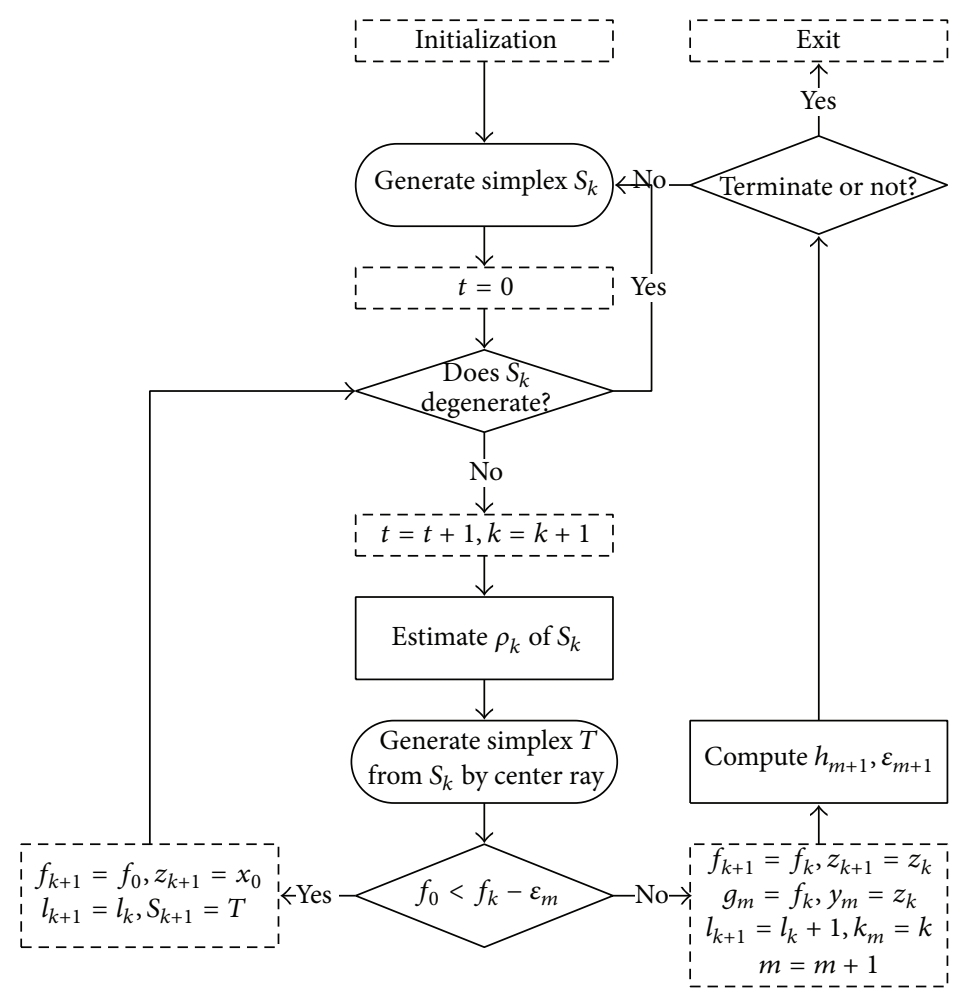

FIgURE 3: The flow of Algorithm 3.

In (24), the gradient can be accurately estimated without additionally calculating the function value. Especially, when the simplex is small or approximate to the extreme point, estimation is very accurate. Furthermore, Hessian matrix, Newton direction as well as the conjugate gradient at each iteration can be approximately estimated according to recent two successive gradients in the process of iterations.

3.1.5. Flow of the Novel Simplex Method. For each simplex, the algorithm looks for a better point along rays emitting from the center $\bar{x}$ to the vertex $x_{i}$ to replace the current vertex $x_{i}$. By continuously replacing the former simplex with a new one, iterations will be repeated until the evolution of the simplex stagnates. Once no better points are worked out or the simplex collapses, a new smaller simplex will be generated at the current optimal point in line with the current estimated descent direction and resume iterations again. The flow of the algorithm is shown in Figure 3.

\section{Algorithm 3.}

Step 1. Initialize the parameters $l_{1}=1, k_{1}=1, m=1, k=1$, $z_{1}=y_{1}, f_{1}=f\left(y_{1}\right), g_{1}=f_{1}, \rho_{1}, \varepsilon_{1}$.

Step 2. Generate simplex $S_{k}$ according to $z_{k}, h_{m},-\rho_{k}$.

Step 2.1. Generate the orthogonal simplex with the side length $h_{m}$ at $z_{k}$ in accordance with Algorithm 1 .

Step 2.2. Sort vertexes $x_{i}$ of simplex $S_{k}$ in ascending order by $f\left(x_{i}\right)$ to form $S_{k}=\left\{x_{0}, x_{1}, \ldots, x_{i}, \ldots, x_{n}\right\}$.
Step 2.3. Set $t=0$.

Step 3. Determine whether $S_{k}$ has degenerated. In case of degeneration, go to Step 2; or else, go to Step 4 .

Step 4. Set $k=k+1, t=t+1$.

Step 5. Estimate the gradient $\rho_{k}$ of the simplex $S_{k}$.

Step 5.1. Compute each side vector $\vec{v}_{i}$ by (22) and construct matrix $V_{k}$.

Step 5.2. Approximately compute the directional derivative in the side vector by (23) and construct the matrix $D=$ $\left[d_{1}, d_{2}, \ldots, d_{n}\right]^{T}$.

Step 5.3. Compute $\rho_{k}$ with (24).

Step 6. Generate simplex $T$ after center ray operations of simplex $S_{k}$.

Step 6.1. Compute $\bar{x}, f(\bar{x})$ and set $i=1$.

Step 6.2. Compute $\mu_{i}$ by (9) or (10), and compute $\widehat{x}_{i}^{e}$ and $f\left(\widehat{x}_{i}^{e}\right)$ with (7) and (8).

Step 6.3. Determine $\mu^{q}$ that satisfies (12), and then compute $\tilde{x}_{i}^{e}, f\left(\widetilde{x}_{i}^{e}\right)$ with (11) to the second detect.

Step 6.4. If $f(\bar{x})>f\left(\widehat{x}_{e}\right)$, choose the best point from $\widetilde{x}_{i}^{e}, \widehat{x}_{i}^{e}$ as a new vertex of simplex $T$ to replace $x_{i}$; otherwise, choose the best point from $x_{i}, \widetilde{x}_{i}^{e}$ as a new vertex of simplex $T$. 


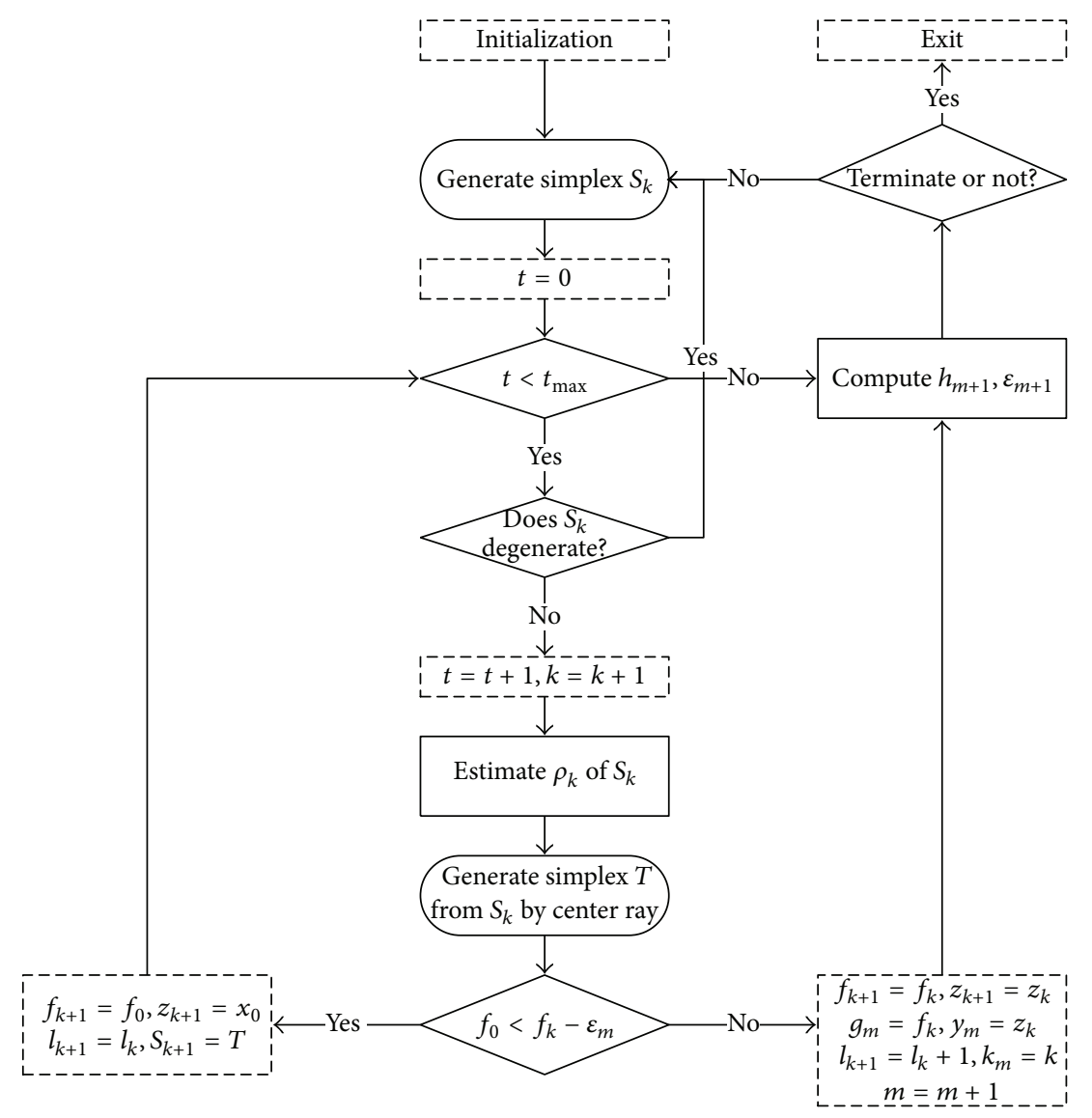

Figure 4: The flow of Algorithm 4.

Step 6.5. If $i<n$, then $i=i+1$ and go to Step 6.2; otherwise, go to Step 6.6.

Step 6.6. Sort vertexes of simplex $T$ in ascending order by their function values.

Step 7. When $f\left(x_{0}\right)<f_{k}-\varepsilon_{m}$, then let $f_{k+1}=f\left(x_{0}\right), z_{k+1}=$ $x_{0}, l_{k+1}=l_{k}, S_{k+1}=T$ and go to Step 3. Otherwise, let $f_{k+1}=$ $f_{k}, z_{k+1}=z_{k}, g_{m}=f_{k}, y_{m}=z_{k}, l_{k+1}=l_{k}+1, k_{m}=k$, $m=m+1$ and go to Step 8 .

Step 8. Compute $h_{m+1}, \varepsilon_{m+1}$ by (25) or (26). If the result satisfies termination conditions, then quit the algorithm; otherwise, go to Step 2 and repeat iterations.

In Algorithm 3, the $m$ th execution of the inner loop from Step 3 to Step 7 generates the subsequence $\left\{z_{k}\right\},\left\{f_{k}\right\}, k_{m} \leq$ $k<k_{m+1}$, which satisfies $f_{k} \leq f_{k-1}-\varepsilon_{m}<f_{k-1}$. As the outer loop from Step 2 to Step 8 repeatedly performs the inner loop, the algorithm generates the infinite sequence $\left\{z_{k}\right\},\left\{f_{k}\right\},\left\{y_{m}\right\},\left\{g_{m}\right\}$, which satisfies $f_{k} \leq f_{k-1}, g_{m} \leq g_{m-1}$.

The center ray only searches along $n+1$ fixed directions. If the direction is not descending, the center ray may fail, and the search will stop for the simplex tends to beinfinitely small. For some ill-conditioned problems, even though search directions are descent, it is theoretically feasible to find optimum value; however, this can be realized only when the step is extremely small. Since such extremely small simplex is likely to collapse or the computation is too slow, it is difficult to realize in practice. Although Algorithm 3 is theoretically convergent, sometimes, it actually tends to converge to saddle point and nonoptimum point in case of complex functions. The performance only can be improved through continuous changes of searching directions. A new simplex will be forced to generate when the number of successive execution of ray operations exceeds certain threshold, which is equivalent to changes of the search direction to some degree. Whereby Algorithm 4 is formed with improvements of Algorithm 3, as shown in Figure 4.

\section{Algorithm 4}

Step 1. Initialize parameters $l_{1}=1, k_{1}=1, m=1, k=1$, $z_{1}=y_{1}, f_{1}=f\left(y_{1}\right), g_{1}=f_{1}, \rho_{1}, \varepsilon_{1}$.

Step 2. Generate simplex $S_{k}$ according to $z_{k}, h_{m},-\rho_{k}$.

Step 2.1. Generate the orthogonal simplex with the side length $h_{m}$ at $z_{k}$ in accordance with Algorithm 1 .

Step 2.2. Sort vertexes $x_{i}$ of simplex $S_{k}$ in ascending order by $f\left(x_{i}\right)$ to form $S_{k}=\left\{x_{0}, x_{1}, \ldots, x_{i}, \ldots, x_{n}\right\}$.

Step 2.3. Set $t=0$. 
Step 3. If $t<t_{\max }$, go to Step 4; otherwise, go to Step 9 .

Step 4. Determine whether $S_{k}$ has degenerated. In case of degeneration, go to Step 2; or else, go to Step 5.

Step 5. Set $k=k+1, t=t+1$.

Step 6. Estimate the gradient $\rho_{k}$ of the simplex $S_{k}$.

Step 6.1. Compute each side vector $\vec{v}_{i}$ by (22) and create the matrix $V_{k}$.

Step 6.2. Approximately compute the directional derivative in the side vector by (23) to construct the matrix $D=$ $\left[d_{1}, d_{2}, \ldots, d_{n}\right]^{T}$.

Step 6.3. Compute $\rho_{k}$ by (24).

Step 7. Generate simplex $T$ after the application of center ray operations of simplex $S_{k}$.

Step 7.1. Compute $\bar{x}, f(\bar{x})$ and let $i=1$.

Step 7.2. Compute $\mu_{i}$ by (9) or (10) and compute $\widehat{x}_{i}^{e}$ and $f\left(\widehat{x}_{i}^{e}\right)$ with (7) and (8).

Step 7.3. Determine $\mu^{q}$ which satisfies (12), compute $\tilde{x}_{i}^{e}, f\left(\tilde{x}_{i}^{e}\right)$ with (11) to the second search.

Step 7.4. If $f(\bar{x})>f\left(\widehat{x}_{e}\right)$, choose the best point from $\tilde{x}_{i}^{e}, \hat{x}_{i}^{e}$ as a new vertex of simplex $T$ to replace $x_{i}$; otherwise, choose the best point from $x_{i}, \widetilde{x}_{i}^{e}$ as a new vertex of $T$.

Step 7.5. If $i<n$, then set $i=i+1$ and go to Step 7.2; otherwise, go to Step 7.6.

Step 7.6. Sort vertexes of simplex $T$ in ascending order by their function values.

Step 8. When $f\left(x_{0}\right)<f_{k}-\varepsilon_{m}$, let $f_{k+1}=f\left(x_{0}\right), z_{k+1}=x_{0}$, $l_{k+1}=l_{k}, S_{k+1}=T$ and go to Step 3. Otherwise, let $f_{k+1}=f_{k}$, $z_{k+1}=z_{k}, g_{m}=f_{k}, y_{m}=z_{k}, l_{k+1}=l_{k}+1, k_{m}=k, m=m+1$ and go to Step 9.

Step 9. Compute $h_{m+1}, \varepsilon_{m+1}$ by (25) or (26). If the result satisfies termination conditions, quit the algorithm; otherwise, go to Step 2 and repeat computation.

Different from Algorithm 3, Algorithm 4 makes restrictions on the times of the repetitive execution of ray operations, denoted as $t$, which is not allowed to surpass the maximum value $t_{\max }$. If $t \geq t_{\max }$, even though ray operations are successful, a new simplex at the current optimal point $z_{k}$ is generated according to the current $\rho_{k}$ so as to change the search direction.

Considering the better performance of reflection operations in the searching process of traditional simplex, in order to further improve the search abilities, a reflection operation will be performed in case of failure of ray operations. Consequently, search directions are more abundant and Algorithm 5 is formed, as shown in Figure 5.
Algorithm 5.

Step 1. Initialize parameters as $l_{1}=1, k_{1}=1, m=1, k=1$, $z_{1}=y_{1}, f_{1}=f\left(y_{1}\right), g_{1}=f_{1}, \rho_{1}, \varepsilon_{1}$.

Step 2. Generate simplex $S_{k}$ according to $z_{k}, h_{m},-\rho_{k}$.

Step 2.1. Generate the orthogonal simplex with the side length $h_{m}$ at $z_{k}$ in accordance with Algorithm 1 .

Step 2.2. Sort vertexes $x_{i}$ of simplex $S_{k}$ in ascending order by $f\left(x_{i}\right)$ to form $S_{k}=\left\{x_{0}, x_{1}, \ldots, x_{i}, \ldots, x_{n}\right\}$.

Step 2.3. Set $t=0$.

Step 3. If $t<t_{\max }$, go to Step 4; otherwise, go to Step 11.

Step 4. Determine whether $S_{k}$ has degenerated. In case of degeneration, go to Step 2; or else, go to Step 5.

Step 5. Set $k=k+1, t=t+1$.

Step 6. Estimate the gradient $\rho_{k}$ of the simplex $S_{k}$.

Step 6.1. Compute each side vector $\vec{v}_{i}$ by (22) to construct matrix $V_{k}$.

Step 6.2. Approximately compute the directional derivative in the side vector by (23), and construct matrix $D=$ $\left[d_{1}, d_{2}, \ldots, d_{n}\right]^{T}$.

Step 6.3. Compute $\rho_{k}$ by (24).

Step 7. Generate simplex $T_{1}$ after the application of center ray operations of simplex $S_{k}$.

Step 7.1. Compute $\bar{x}, f(\bar{x})$ and set $i=1$.

Step 7.2. Determine $\mu_{i}$ by (9) or (10) and compute $\hat{x}_{i}^{e}$ and $f\left(\widehat{x}_{i}^{e}\right)$ by $(7)$ and $(8)$.

Step 7.3. Determine $\mu^{q}$ which satisfies (12) and compute $\tilde{x}_{i}^{e}, f\left(\tilde{x}_{i}^{e}\right)$ by (11) to the second detection.

Step 7.4. If $f(\bar{x})>f\left(\widehat{x}_{e}\right)$, choose the best point from $\tilde{x}_{i}^{e}, \widehat{x}_{i}^{e}$ as a new vertex of simplex $T_{1}$ to replace $x_{i}$; otherwise, choose the best point from $x_{i}, \tilde{x}_{i}^{e}$ as a new vertex of simplex $T_{1}$.

Step 7.5. If $i<n$, then set $i=i+1$ and go to Step 7.2; otherwise, go to Step 7.6.

Step 7.6. Sort vertexes of simplex $T_{1}$ in ascending order by their function value.

Step 8. When $f\left(x_{0}\right)<f_{k}-\varepsilon_{m}$, set $f_{k+1}=f\left(x_{0}\right), z_{k+1}=x_{0}$, $l_{k+1}=l_{k}, S_{k+1}=T_{1}$ and go to Step 3. Otherwise, go to Step 9.

Step 9. Form novel simplex $T_{2}$ after the reflection of simplex $T_{1}$.

Step 9.1. Compute $\bar{x}^{\prime}$ by (13).

Step 9.2. The reflection coefficient $\alpha$ is computed by (16) or (17) and $\hat{x}^{r}$ and $f\left(\hat{x}^{r}\right)$ are computed by (14). 


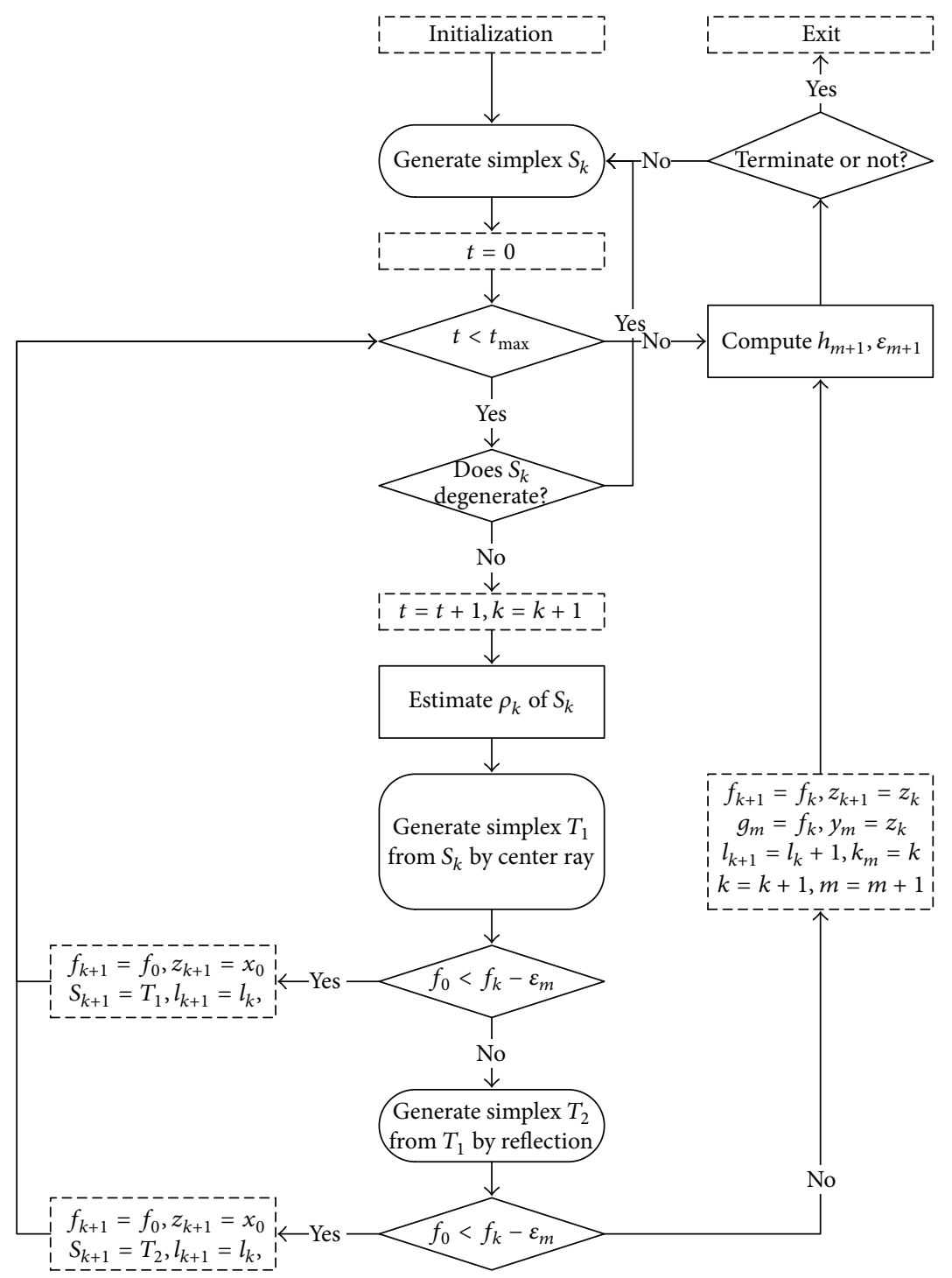

Figure 5: The flow of Algorithm 5.

Step 9.3. Determine $\alpha_{q}$ which satisfies (19) or (20), and then solve $\tilde{x}^{r}$ by (18) and compute $f\left(\tilde{x}^{r}\right)$.

Step 9.4. Determine reflection point $x^{r}$ by (21). If $f\left(x^{r}\right)<$ $f\left(x_{n}\right)$, then $x_{n}$ is replaced with $x^{r}$ to form new simplex $T_{2}$ and sort vertexes of $T_{2}$ in ascending order by function value.

Step 10. When $f\left(x_{0}\right)<f_{k}-\varepsilon_{m}$, set $f_{k+1}=f\left(x_{0}\right), z_{k+1}=x_{0}$, $l_{k+1}=l_{k}, S_{k+1}=T_{2}$ and go to Step 3. Otherwise, set $f_{k+1}=f_{k}$, $z_{k+1}=z_{k}, g_{m}=f_{k}, y_{m}=z_{k}, l_{k+1}=l_{k}+1, k_{m}=k, m=m+1$ and go to Step 11 .

Step 11. Compute $h_{m+1}, \varepsilon_{m+1}$ by (25) or (26). If the result satisfies termination conditions, quit the algorithm; otherwise, go to Step 2 to repeatedly do loop computations.

Similarly, as for Algorithm 5, Step 3 to Step 10 construct inner loop while Step 2 to Step 11 form the outer loop.
Algorithms 3, 4, and 5 all have two layers of loops. The sequence $\left\{z_{k}\right\}$ is composed of optimal points of simplex $S_{k}$. The $m$ th execution of the inner loop generates the finite sequence $\left\{z_{k}\right\}, k_{m} \leq k<k_{m+1}$, which satisfies $f\left(z_{k}\right) \leq$ $f\left(z_{k-1}\right)-\varepsilon_{m}<f\left(z_{k-1}\right)$. The outer loop will be repeatedly executed, which may generate the infinite sequence $\left\{z_{k}\right\}$ satisfying $f\left(z_{k+1}\right) \leq f\left(z_{k}\right)$. That is to say, the sequence $f\left(z_{k}\right)$ generated by the algorithm must be nonincreasing. The sequence $\left\{y_{m}\right\}$ is composed of best vertexes of the simplex when both ray operations and reflection operations fail, which satisfies $f\left(y_{m+1}\right) \leq f\left(y_{m}\right)$. Upon the failure of ray and reflection operations, a new smaller orthogonal simplex will be generated. The algorithm can ensure the current minimum point to be one of the vertexes of the new simplex. Besides, it also ensures that the current descent direction is parallel to an edge of the new simplex. The generation of a new smaller simplex achieves better effect than shrinkage operations, which can reduce the possibility of the simplex degeneration. $l_{k}$ indicates 
the number of simplexes generation till the $k$ th iteration. $k_{m}$ means that the $m$ th simplex generation occurs at the $k$ th iteration. $h_{m}$ means the initial edge length of the $m$ th generated simplex. For the sake of enhancing the efficiency, $h_{m}$ can increase in the process of iterations. However, it is certain that there are decreasing subsequences in $\left\{h_{m}\right\} . \varepsilon_{m}$ is the control error, which is a monotonically decreasing sequence in the process of iterations. In order to converge, $h_{m}, \varepsilon_{m}$ must satisfy $\lim _{m \rightarrow \infty} h_{m}=0$ and $\lim _{m \rightarrow \infty}\left(\varepsilon_{m} / h_{m}\right)=0$. In the paper, $h_{m}, \varepsilon_{m}$ are solved by (25) or (26):

$$
\begin{aligned}
& h_{m}=h_{m-1} \lambda_{h}, \\
& \varepsilon_{m}=\varepsilon_{m-1} \lambda_{\varepsilon}, \\
& \lambda_{h}= \begin{cases}\dot{\lambda}_{h} & t \geq t_{\max } \\
\ddot{\lambda}_{h} & \left(\left\|\rho_{m}\right\| \leq \rho_{\text {nei }} \vee t \leq t_{\text {dec }}\right) \wedge h_{m-1}>h_{\text {small }} \\
\dddot{\lambda} & \text { others, }\end{cases} \\
& \lambda_{\varepsilon}= \begin{cases}\ddot{\lambda}_{\varepsilon} & \left\|\rho_{m}\right\| \leq \rho_{\text {nei }} \\
\dddot{\lambda} & \left\|\rho_{m}\right\|>\rho_{\text {nei }} .\end{cases}
\end{aligned}
$$

The variation of $h_{m}$ has important influences on the performance of the algorithm. The rate of change of $h_{m}, \varepsilon_{m}$ must satisfy $1<\dot{\lambda}_{h}, 0<\ddot{\lambda}_{h}<\dddot{\lambda}_{h}<1,0<\ddot{\lambda}_{\varepsilon}<\dddot{\lambda}_{\varepsilon}<1$ in the paper.

Theorem 6. As for Algorithm 3, assuming the sequence $z_{k}$ is bounded, the objective function $f(x)$ is continuously differentiable and $f^{\prime}(x)$ is Lipschitz in any bounded region of $R^{n}$; then, $\left\{y_{m}\right\}$ converges at the stationary point of $f(x)$.

Proof. It can be learned from Theorem 2 that the search direction $u_{i}=x_{i}-\bar{x}, i=0, \ldots, n$, is positive bases. From the flow of the algorithms, the finite sequence $\left\{f\left(z_{k}\right)\right\}, k_{m} \leq$ $k<k_{m+1}$, generated by the inner loop is monotonically decreasing as $f\left(z_{k}\right)>f\left(z_{k+1}\right), k_{m} \leq k<k_{m+1}$. Upon the exiting from the inner loop, it is certain $f\left(z_{k+1}\right)+\varepsilon_{m} \geq$ $f\left(z_{k}\right)$ will hold. So $f\left(z_{k}\right) \geq f\left(z_{k+1}\right)$ will be obtained by the algorithms. Similar to the frame of Algorithms 3.1 and 3.2 described in Coope and Price (2000), $z_{k}$ constitutes a quasiframe [11]. Comparing those two algorithms in Coope and Price (2000) with Algorithms 3, 4, and 5 in the paper, the algorithm proposed in the paper can be regarded as a concrete instance of Algorithms 3.1 and 3.2 in Coope and Price (2000). The side vectors $x_{i}-x_{0}, i=1, \ldots, n$, and $\bar{x}-x_{0}$ of the simplex are regarded as positive bases in Coope and Price (2000) [11]. However, $x_{i}-\bar{x}, i=0, \ldots, n$, are taken as positive bases in the paper. Algorithms in Coope and Price (2000) have same convergence properties as algorithms in the paper, whose convergence is proved in Coope and Price (2000) in details [11]. Compared with Algorithm 3, some acceleration methods are adopted in Algorithms 4 and 5, so their convergence is similar to Algorithm 3.

3.2. Experimental Verification of the Novel Simplex Algorithm. Schwefel and Rosenbrock functions are taken as benchmarks to test the performance of the improved simplex algorithm
[12]. In the experiments, the initial point at each computation is all $x_{0}=[50, \ldots, 50]^{T}$. Once the number of iterations exceeds the predetermined maximum value or $h_{m}$ approaches zero or the solution is obtained, the algorithm will immediately be terminated. $\mu_{\text {outer }}$ and $\mu_{\text {inner }}$ are computed by (9) and, respectively, set $\mu_{\text {outer }}=1.2, \mu_{\text {inner }}=0.9, \mu^{e}=1.25, \mu^{o c}=$ $0.92, \mu^{i c}=1.06$, and $\mu^{s}=0.4$. Besides, reflection coefficient $\alpha$ is computed by (16) and set $\alpha^{\text {forward }}=1.3, \alpha^{\text {reverse }}=-2.3$, $\alpha^{f e}=1.25, \alpha^{f c}=0.7, \alpha^{f s}=-0.4, \alpha^{r e}=1.25, \alpha^{r c}=0.72$, and $\alpha^{r s}=0.15$. In the experiments, the other parameters are $h_{0}=5, \varepsilon_{0}=0.2, \rho_{\text {nei }}=0.1, \ddot{\lambda}_{\varepsilon}=0.5, \dddot{\lambda}_{\varepsilon}=0.8, \dot{\lambda}_{h}=1.5$. Assuming that actual number of iterations is $\Omega_{1}$, the number of function computation is $\Omega_{2}$ and the optimal solution is $f(\widehat{x})$, then $\Omega_{3}=\left\|\widehat{x}-x_{0}\right\| / \Omega_{1}, \Omega_{4}=\left|f(\widehat{x})-f\left(x_{0}\right)\right| / \Omega_{1}$, $\Omega_{5}=\left\|\widehat{x}-x_{0}\right\| / \Omega_{2}, \Omega_{6}=\left|f(\widehat{x})-f\left(x_{0}\right)\right| / \Omega_{2}, \Omega_{7}=\left\|\widehat{x}-x^{*}\right\|$ can be utilized to evaluate the performance of the algorithm. Smaller $\Omega_{1}, \Omega_{2}, \Omega_{7}$ and larger $\Omega_{3}, \Omega_{4}, \Omega_{5}, \Omega_{6}$ indicate the performance of the algorithm is better.

Price and Coope (2002) has improved NM Simplex method and introduced ghost simplex [13]. The algorithm revised by PRICE is tested in the paper. It is concluded that either Price's algorithm or NM Simplex method can hardly solve when the dimension of Rosenbrock functions is more than 20. In other words, in solving high-dimension problems, N M Simplex method has presented very poor effect and the algorithm proposed by PRICE also makes little improvement. Taking 5-dimensional Schwefel function as the benchmark, the algorithm is terminated once $\Omega_{7}=4 e-5$. Then, the results of this paper are compared with those of Price's method. Some results are listed in Table 2 . In order to verify the performance of the algorithm proposed in the paper in highdimension optimization problems, 90-dimensional Schwefel function and 45-dimensional Rosenbrock function are solved by Algorithms 4, 5, and 7 (Figure 6). Some results are shown in Tables 3 8. In order to compare effects of all algorithms, average values are totaled in Tables $3 \sim 8$, as shown in Figure 5. The experiment proves that the effect and performance of improved simplex algorithm proposed in the paper is much better than that of traditional simplex methods.

In terms of optimization of low-dimensional functions, though the algorithm proposed by the paper and by PRICE can solve such problems, the improved simplex method in the paper demonstrates higher efficiency. It can be known from Table 2 that the algorithm proposed in the paper computes much rapidly although the times of object function computation by Algorithms 3,4, and 5 is more than that of Price's algorithm. The object function is calculated twice every detection in iteration, so not only $\Omega_{2}$ of the algorithm proposed by the paper may be great than that of other simplex method but also $\Omega_{5}, \Omega_{6}$ of algorithm in my paper may be less than that of other simplex method under low dimensional circumstance. In addition, as Price's algorithm and the traditional simplex algorithm are quite inefficient in high-dimension optimization problems, they can hardly solve any problem as long as the number of dimensions is over 20. In comparison, the algorithm proposed in the paper still behaves more effectively in solving high-dimension problems. It indicates that the improved simplex method, 
TABLE 2: Comparison between the algorithm proposed in the paper and Price.

\begin{tabular}{lccccccc}
\hline Algorithm & $f(\widehat{x})$ & $\Omega_{1}$ & $\Omega_{2}$ & $\Omega_{3}$ & $\Omega_{4}$ & $\Omega_{5}$ & $\Omega_{6}$ \\
\hline Price & $1.361376 E-09$ & 2457 & 3569 & 0.045504 & 55.96256 & 0.031326 & 38.5262 \\
Algorithm 3 & $2.161297 E-09$ & 602 & 11226 & 0.18572 & 228.4053 & 0.009959 & 12.24835 \\
Algorithm 4 & $1.703474 E-09$ & 526 & 11305 & 0.212554 & 261.4068 & 0.00989 & 12.16276 \\
Algorithm 5 & $1.314375 E-09$ & 448 & 9220 & 0.249561 & 306.9196 & 0.012126 & 14.91323 \\
\hline
\end{tabular}
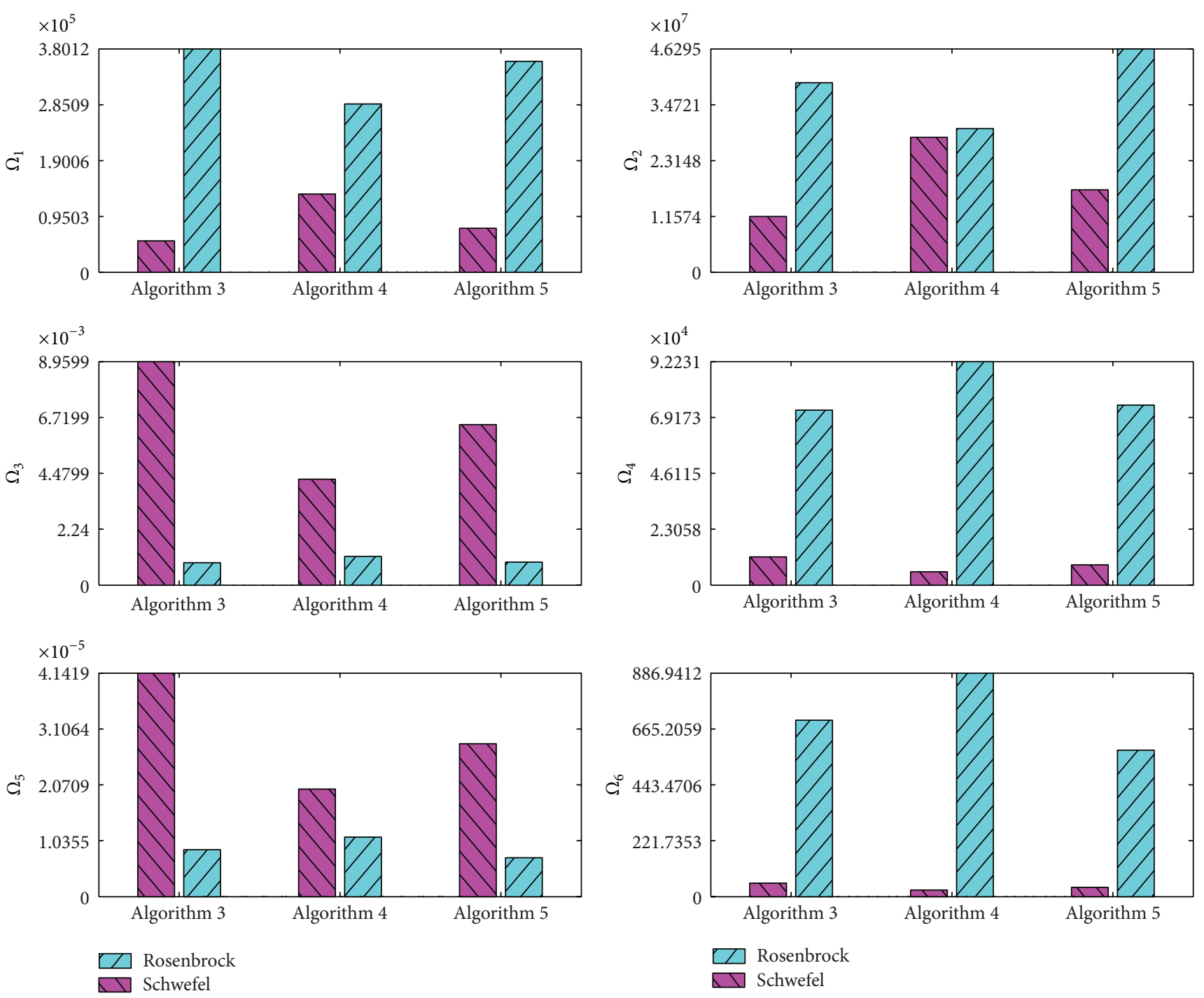

FIgURE 6: Comparison of different effects of Algorithms 3, 4, and 5.

which can update several vertexes at each iteration by center ray operations, is effective and efficient.

With respect to the performance, generally, Algorithm 5 is the best, while Algorithm 4 is better than Algorithm 3. For both Rosenbrock function and Schwefel function, Algorithm 5 can obtain the smallest $\Omega_{1}, \Omega_{2}$ and the largest $\Omega_{3}, \Omega_{4}, \Omega_{5}, \Omega_{6}$; this implies that increasing of search directions can greatly improve the efficiency. $\Omega_{1}, \Omega_{2}$ of
Algorithm 4 are smaller than those of Algorithm 3, which indicates that the former costs less in computation. In general, $\Omega_{3}, \Omega_{4}, \Omega_{5}, \Omega_{6}$ of Algorithm 3 is smaller than that of Algorithm 4, which reflects that efficiency and speed of Algorithm 4 is higher than that of Algorithm 3.

The parameters of the algorithm, especially the parameters controlling changes of $h_{m}$ have great influence on the performance of the algorithm. According to the experiment, 
TABLE 3: Parts of results of solving 90-dimensional Schwefel function by Algorithm 3.

\begin{tabular}{lccccccccc}
\hline$\ddot{\lambda}_{h}$ & $\dddot{\lambda}_{h}$ & $f(\widehat{x})$ & $\Omega_{1}$ & $\Omega_{2}$ & $\Omega_{3}$ & $\Omega_{4}$ & $\Omega_{5}$ & $\Omega_{6}$ \\
\hline 0.9 & 0.99 & $6.96 E-08$ & 60683 & 13153005 & 0.007817 & 10178.51 & $3.61 E-05$ & 46.9598 & 0.000516 \\
0.9 & 0.98 & $7.37 E-08$ & 53731 & 11575442 & 0.008828 & 11495.46 & $4.1 E-05$ & 53.35973 & 0.000465 \\
0.9 & 0.975 & $8.98 E-08$ & 48455 & 10472012 & 0.009789 & 12747.14 & $4.53 E-05$ & 58.98222 & 0.000593 \\
0.85 & 0.99 & $6.5 E-08$ & 59400 & 12882921 & 0.007986 & 10398.36 & $3.68 E-05$ & 47.94429 & 0.000504 \\
0.85 & 0.98 & $7.38 E-08$ & 49668 & 10766210 & 0.00955 & 12435.82 & $4.41 E-05$ & 57.37047 & 0.000429 \\
0.85 & 0.975 & $8.98 E-08$ & 48455 & 10472012 & 0.009789 & 12747.14 & $4.53 E-05$ & 58.98222 & 0.000593 \\
\hline
\end{tabular}

TABLE 4: Parts of results of solving 45-dimensional Rosenbrock function by Algorithm 3.

\begin{tabular}{lccccccccc}
\hline$\ddot{\lambda}_{h}$ & $\ddot{\lambda}_{h}$ & $f(\widehat{x})$ & $\Omega_{1}$ & $\Omega_{2}$ & $\Omega_{3}$ & $\Omega_{4}$ & $\Omega_{5}$ & $\Omega_{6}$ \\
\hline 0.9 & 0.995 & $3.32 E-09$ & 299760 & 30953026 & 0.001097 & 88107.5 & $1.06 E-05$ & 853.2641 & 0.000115 \\
0.9 & 0.985 & $3.32 E-09$ & 446429 & 46260909 & 0.000736 & 59160.82 & $7.11 E-06$ & 570.9163 & 0.000115 \\
0.85 & 0.995 & $3.32 E-09$ & 316215 & 32404535 & 0.001039 & 83522.62 & $1.01 E-05$ & 815.0435 & 0.000115 \\
0.85 & 0.985 & $3.32 E-09$ & 454935 & 47117032 & 0.000723 & 58054.68 & $6.98 E-06$ & 560.5426 & 0.000115 \\
0.8 & 0.995 & $3.32 E-09$ & 303212 & 31274881 & 0.001084 & 87104.42 & $1.05 E-05$ & 844.483 & 0.000115 \\
0.8 & 0.985 & $3.32 E-09$ & 460166 & 47576852 & 0.000714 & 57394.74 & $6.91 E-06$ & 555.1251 & 0.000115 \\
\hline
\end{tabular}

TABle 5: Parts of results of solving 90-dimensional Schwefel function by Algorithm 4.

\begin{tabular}{cccccccccc}
\hline$\ddot{\lambda}_{h}$ & $\ddot{\lambda}_{h}$ & $f(\widehat{x})$ & $\Omega_{1}$ & $\Omega_{2}$ & $\Omega_{3}$ & $\Omega_{4}$ & $\Omega_{5}$ & $\Omega_{6}$ \\
\hline 0.9 & 0.99 & $8.34 E-08$ & 67054 & 14486323 & 0.007074 & 9211.419 & $3.27 E-05$ & 42.63763 & 0.000567 \\
0.9 & 0.98 & $8.78 E-08$ & 130898 & 27575059 & 0.003624 & 4718.655 & $1.72 E-05$ & 22.39932 & 0.000589 \\
0.9 & 0.975 & $7.08 E-08$ & 194194 & 40244951 & 0.002443 & 3180.647 & $1.18 E-05$ & 15.34758 & 0.00052 \\
0.85 & 0.99 & $8.75 E-08$ & 73219 & 15845655 & 0.006478 & 8435.823 & $2.99 E-05$ & 38.97993 & 0.000584 \\
0.85 & 0.98 & $8.52 E-08$ & 138163 & 29067816 & 0.003433 & 4470.535 & $1.63 E-05$ & 21.24902 & 0.00058 \\
0.85 & 0.975 & $4.68 E-08$ & 195527 & 40501017 & 0.002426 & 3158.963 & $3.23 E-05$ & 15.25054 & 0.000505 \\
\hline
\end{tabular}

TABLE 6: Parts of results of solving 45-dimensional Rosenbrock function by Algorithm 4.

\begin{tabular}{lccccccccc}
\hline$\ddot{\lambda}_{h}$ & $\ddot{\lambda}_{h}$ & $f(\hat{x})$ & $\Omega_{1}$ & $\Omega_{2}$ & $\Omega_{3}$ & $\Omega_{4}$ & $\Omega_{5}$ & $\Omega_{6}$ \\
\hline 0.9 & 0.995 & $3.32 E-09$ & 285070 & 29702517 & 0.001153 & 92647.79 & $1.11 E-05$ & 889.1875 & $1.72 E-05$ \\
0.9 & 0.985 & $3.32 E-09$ & 287837 & 29884227 & 0.001142 & 91757.16 & $1.10 E-05$ & 883.7808 & $1.72 E-05$ \\
0.85 & 0.995 & $3.32 E-09$ & 283558 & 29541881 & 0.001159 & 93141.81 & $1.11 E-05$ & 894.0225 & $1.72 E-05$ \\
0.85 & 0.985 & $3.32 E-09$ & 285806 & 29672230 & 0.00115 & 92409.21 & $1.11 E-05$ & 890.0951 & $1.72 E-05$ \\
0.8 & 0.995 & $3.32 E-09$ & 287742 & 29952651 & 0.001142 & 91787.45 & $1.10 E-05$ & 881.7619 & $1.72 E-05$ \\
0.8 & 0.985 & $3.32 E-09$ & 288202 & 29917444 & 0.001141 & 91640.95 & $1.10 E-05$ & 882.7995 & $1.72 E-05$ \\
\hline
\end{tabular}

TABLE 7: Parts of results of solving 90-dimensional Schwefel function by Algorithm 5.

\begin{tabular}{lccccccccc}
\hline$\ddot{\lambda}_{h}$ & $\ddot{\lambda}_{h}$ & $f(\widehat{x})$ & $\Omega_{1}$ & $\Omega_{2}$ & $\Omega_{3}$ & $\Omega_{4}$ & $\Omega_{5}$ & $\Omega_{6}$ \\
\hline 0.9 & 0.99 & $4.06 E-08$ & 74783 & 21242674 & 0.006343 & 8259.397 & $2.23 E-05$ & 29.07649 & 0.0004 \\
0.9 & 0.98 & $7.08 E-08$ & 75482 & 16388224 & 0.006284 & 8182.911 & $2.89 E-05$ & 37.68941 & 0.000516 \\
0.9 & 0.975 & $8.86 E-08$ & 81503 & 17609179 & 0.00582 & 7578.402 & $2.69 E-05$ & 35.07617 & 0.000581 \\
0.85 & 0.99 & $7.57 E-08$ & 59474 & 13368291 & 0.007976 & 10385.42 & $3.55 E-05$ & 46.20355 & 0.000542 \\
0.85 & 0.98 & $7.50 E-08$ & 72768 & 15772677 & 0.006519 & 8488.106 & $3.01 E-05$ & 39.16028 & 0.000532 \\
0.85 & 0.975 & $2.38 E-08$ & 84097 & 18157608 & 0.00564 & 7344.644 & $1.42 E-05$ & 34.01673 & 0.000611 \\
\hline
\end{tabular}


TABLE 8: Parts of results of solving 45-dimensional Rosenbrock function by Algorithm 5.

\begin{tabular}{lccccccccc}
\hline$\ddot{\lambda}_{h}$ & $\ddot{\lambda}_{h}$ & $f(\hat{x})$ & $\Omega_{1}$ & $\Omega_{2}$ & $\Omega_{3}$ & $\Omega_{4}$ & $\Omega_{5}$ & $\Omega_{6}$ \\
\hline 0.9 & 0.995 & $3.32 E-09$ & 391063 & 52265101 & 0.000841 & 67536.7 & $6.29 E-06$ & 505.3297 & $1.72 E-05$ \\
0.9 & 0.985 & $3.32 E-09$ & 323520 & 40051422 & 0.001016 & 81636.7 & $8.21 E-06$ & 659.4299 & $1.72 E-05$ \\
0.85 & 0.995 & $3.32 E-09$ & 394208 & 52604873 & 0.000834 & 66997.89 & $6.25 E-06$ & 502.0658 & $1.72 E-05$ \\
0.85 & 0.985 & $3.33 E-09$ & 323368 & 39892530 & 0.001016 & 81675.07 & $8.24 E-06$ & 662.0564 & $1.72 E-05$ \\
0.8 & 0.995 & $3.34 E-09$ & 395380 & 52745863 & 0.000831 & 66799.3 & $6.23 E-06$ & 500.7237 & $1.72 E-05$ \\
0.8 & 0.985 & $3.33 E-09$ & 325750 & 40212144 & 0.001009 & 81077.84 & $8.17 E-06$ & 656.7943 & $1.72 E-05$ \\
\hline
\end{tabular}

it is usually possible for the algorithm to get good effects when main parameters are within the following ranges:

$$
\begin{aligned}
& \mu_{\text {inner }} \in(0.5,1) \text {, } \\
& \mu_{\text {outer }} \in(1.2,2.2) \text {, } \\
& \mu^{e} \in(1.1,1.6), \\
& \mu^{o c} \in(0.5,0.8), \\
& \mu^{i c} \in(1.1,1.8), \\
& \mu^{s} \in(0.1,0.5), \\
& \dot{\lambda}_{h} \in(1.5,3) \text {, } \\
& \ddot{\lambda}_{h} \in(0.5,0.95) \text {, } \\
& \dddot{\lambda}_{h} \in(0.95,0.999) \text {, } \\
& t_{\text {max }} \in(6,16) \text {, } \\
& \rho_{\text {nei }} \in(0.1,0.2), \\
& h_{\text {small }} \in(0.5,0.05) \text {, } \\
& h_{0} \in(0.5,5) \text {, } \\
& \ddot{\lambda}_{\varepsilon} \in(0.5,0.95) \text {, } \\
& \dddot{\lambda}_{\varepsilon} \in(0.8,0.9) \text {, } \\
& \varepsilon_{0} \in(0.2,0.5), \\
& \alpha^{\text {forward }} \in(0.5,2.5) \text {, } \\
& \alpha^{\text {reverse }} \in(-1.1,-3) \text {, } \\
& \alpha_{\text {init }}^{\text {forward }} \in(2,3), \\
& \alpha_{\text {end }}^{\text {forward }} \in(0.3,0.6) \text {, } \\
& \alpha_{\text {init }}^{\text {reverse }} \in(-2.5,-3.5) \text {, } \\
& \alpha_{\text {end }}^{\text {reverse }} \in(-1.1,-1.5) \text {, } \\
& \alpha^{f e} \in(1.2,2.5), \\
& \alpha^{f c} \in(0.2,0.8),
\end{aligned}
$$

$$
\begin{aligned}
& \alpha^{f_{s}} \in(-2,-0.4), \\
& \alpha^{r e} \in(1.2,2.5), \\
& \alpha^{r c} \in(0.3,0.9), \\
& \alpha^{r s} \in(0,0.4) .
\end{aligned}
$$

\section{Combinatorial Optimization of Aircraft Maintenance Tasks}

4.1. Analysis of the Combinatorial Optimization of Aircraft Maintenance Tasks. Taking the actual requirements of the combination of aircraft maintenance tasks into consideration, a constraint optimization model of the combination of maintenance tasks is created on the basis of model (3). Algorithms 3,4 , and 5 in the paper can be applied to solve the optimization model, and subsequently the approximate optimal maintenance work packages are obtained [14].

4.1.1. Combinatorial Optimization Model of Aircraft Maintenance Tasks. The basic properties of the $j$ th preventive maintenance task of aircraft includes ATA code, maintenance interval, zone, check and, man-hours, respectively, denoted as

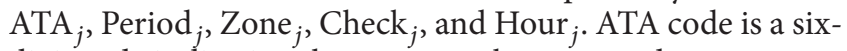
digit code indicating the system, subsystem and components of the aircraft. The last two digits of ATA code are not uniformly standardized, so only the preceding four codes are adopted in the paper. Being interval-valued, maintenance interval refers to the floating range of the maintenance cycle and is denoted as Period $_{j}=\left[\underline{P}_{j}, \bar{P}_{j}\right]$. In terms of ATA standards, the structure and the space of a civil aircraft are usually divided into several zones. Every task is executed in some zones. There are great similarities among maintenance tasks in adjacent zones. In line with MSG-3, there are five types of checks, namely, LU/SV, OP/VC, IN/FC, RS, and DS. If we define set $\mathfrak{I}=\{\mathrm{LU} / \mathrm{SV}, \mathrm{OP} / \mathrm{VC}, \mathrm{IN} / \mathrm{FC}, \mathrm{RS}, \mathrm{DS}\}$, then $\mathrm{Check}_{j} \in \mathfrak{\Im}$. Man-hours represents how long time execution of the maintenance task need, which can be utilized to measure the size of the work package. $\mathrm{ATA}_{j}$, Period $_{j}, Z_{\text {Zne }}$, and $\mathrm{Check}_{j}$ are utilized to measure similarities between maintenance tasks. However, roles and influences of the four properties on similarity evaluation are very different. Weights $w_{\text {period }}, w_{\text {zone }}, w_{\text {check }}$, and $w_{\text {ATA }}$ are, respectively, quantified those differences. 
Maintenance interval is interval-valued. It is necessary to redefine its distance and inner product to adapt to model (3) $[15,16]$. Supposing that set $V$ is composed of interval-valued $[a, b], a, b \in R^{n}$, and $s_{1}=\left[a_{1}, b_{1}\right] \in V, s_{2}=\left[a_{2}, b_{2}\right] \in V, k \in R$, if addition is defined as $s_{1}+s_{2}=\left[a_{1}+a_{2}, b_{1}+b_{2}\right]$ and the scalar multiplication is defined as $k s_{1}=k\left[a_{1}, b_{1}\right]=\left[k a_{1}, k b_{1}\right]$, it can be proved that $V$ is the linear space on $R$ and the zero element is $[0,0]$. In addition, if the inner product is defined as $\left\langle s_{1}, s_{2}\right\rangle=\left\langle\left[a_{1}, b_{1}\right],\left[a_{2}, b_{2}\right]\right\rangle=a_{1}^{T} a_{2}+b_{1}^{T} b_{2}, V$ is proved to be the inner product space. Hence, the distance between $s_{1}$ and $s_{2}$ can be defined as $d_{s_{1} s_{2}}^{2}=\left\langle\left[a_{1}, b_{1}\right]-\left[a_{2}, b_{2}\right],\left[a_{1}, b_{1}\right]-\right.$ $\left.\left[a_{2}, b_{2}\right]\right\rangle=\left(a_{1}-a_{2}\right)^{T}\left(a_{1}-a_{2}\right)+\left(b_{1}-b_{2}\right)^{T}\left(b_{1}-b_{2}\right)$ which is in consistent with the definition of the city block distance $[17,18]$. Obviously, it also meets the requirements of metric distance. Consequently, the distance between $\operatorname{Period}_{i}$ and Period $_{j}$ is defined as $d_{\text {Period }_{i}}^{2}$ Period $_{j}=\left(\underline{P}_{i}-\underline{P}_{j}\right)^{2}+\left(\bar{P}_{i}-\bar{P}_{j}\right)^{2}$.

Check merely involves several discrete values. So the measurement of the similarity or distance is usually specified subjectively from expertise. After detailed investigations and comprehensive researches, similarities of those checks are summarized in Table 9 . Table 9 develops the similarity matrix $\left[\mathfrak{\Im}_{i j}\right]_{5 \times 5}$ of Check $_{i}$ and Check $_{j}$; thus, the distance matrix can be obtained as $d_{\text {Check }_{i} \text {, } \text { Check }_{j}}^{2}=1-\Im_{i j}$ [19].

Zones are symbolized by digital symbols from 100 to 800 . These zone codes can be regarded as digits to measure similarities although they are not numeric values in the real sense. Estimating the distance between two zones by the common numerical computation can approximately meet the requirements of engineering. Suppose that the $i$ th task associates with $\left|Z_{\text {Zone }}\right|$ zones, denoted as Zone $e_{i}=\left\{\mathrm{CZ}_{i, 1}, \ldots\right.$, $\left.\mathrm{CZ}_{i,\left|Z_{\text {Zne }}\right|}\right\}$, and the $j$ th task involves $\left|Z_{\text {Zne }}\right|$ zones, expressed as Zone ${ }_{j}=\left\{\mathrm{CZ}_{j, 1}, \ldots, \mathrm{CZ}_{j, \mid Z_{\text {Zone }}}\right\}$; then, the distance between Zone $_{i}$ and Zone Z $_{j}$ is defined as $d_{\text {Zone }_{i}, \text { Zone }_{j}}^{2}=(1 /$ $\mid$ Zone $_{i}||$ Zone $\left._{j} \mid\right) \sum_{m=1}^{\mid \text {Zone }_{i} \mid} \sum_{n=1}^{\left|Z_{j} e_{j}\right|}\left(\mathrm{CZ}_{i, m}-\mathrm{CZ}_{j, n}\right)^{2}$.
Similar to zone codes, it is acceptable in practice to evaluate similarities of ATA codes by numerical computations if they are treated as numerical values. Thus, the distance between $\mathrm{ATA}_{i}$ and $\mathrm{ATA}_{j}$ is defined as $d_{\mathrm{ATA}_{i}, \mathrm{ATA}_{j}}^{2}=\left\langle\mathrm{ATA}_{i}-\right.$ $\left.\operatorname{ATA}_{j}, \operatorname{ATA}_{i}-\operatorname{ATA}_{j}\right\rangle$.

Supposing the $i$ th maintenance work package is Package ${ }_{i}$, man-hours of the package can be computed by (28) if maintenance tasks are executed in sequence. Its man-hours shall be computed by (29) if maintenance tasks are performed concurrently. To realize the successful execution of maintenance tasks, the workload of the maintenance work package must meet the requirement of actual maintenance capability, namely, $\mathrm{PH}_{i}<\mathrm{PH}_{\max }$, where $\mathrm{PH}_{\max }$ indicates the upper limit of every work package man-hours, which is required by maintenance conditions:

$$
\begin{aligned}
\mathrm{PH}_{i} & =\sum_{j \in \text { Package }_{i}} \text { Hour }_{j}, \\
\mathrm{PH}_{i} & =\max _{j \in \text { Package }_{i}} \text { Hour }_{j} .
\end{aligned}
$$

To satisfy requirements of the reliability and economy, when those work packages are executed, maintenance intervals of work packages should be acceptable to all maintenance tasks in the package. Namely, there shall be a public interval among the intersection of all tasks in the package. If the maintenance interval $P P_{i}$ of the $i$ th maintenance work package Package $_{i}$ is defined as (30), then $\widetilde{P}_{i}-\underset{\sim}{P} \geq 0, \forall i$ is required:

$$
P P_{i}=\left[{\underset{\sim}{P}}_{i}, \widetilde{P}_{i}\right]=\underset{j \in \text { Package }_{i}}{\bigcap}\left[\underline{P}_{j}, \bar{P}_{j}\right]
$$

Correspondingly, the combinatorial optimization model of the maintenance task will be

$$
\begin{aligned}
& J_{m}(U)=w_{\text {period }} \sum_{i=1}^{c} \sum_{j=1}^{n} u_{i j}^{m}\left(\underline{P}_{j}^{2}+\bar{P}_{j}^{2}-\frac{2 \sum_{l=1}^{n} u_{i l}^{m}\left(\underline{P}_{j} \underline{P}_{l}+\bar{P}_{j} \bar{P}_{l}\right)}{\sum_{k=1}^{n} u_{i k}^{m}}+\frac{\sum_{p=1}^{n} \sum_{q=1}^{n} u_{i p}^{m} u_{i q}^{m}\left(\underline{P}_{p} \underline{P}_{q}+\bar{P}_{p} \bar{P}_{q}\right)}{\left(\sum_{k=1}^{n} u_{i k}^{m}\right)^{2}}\right) \\
& \quad+w_{\text {zone }} \sum_{i=1}^{c} \sum_{j=1}^{n} u_{i j}^{m} d_{\text {Zone }_{i}, \text { Zone }_{j}}^{2}+w_{\text {ATA }} \sum_{i=1}^{c} \sum_{j=1}^{n} u_{i j}^{m}\left(\mathrm{ATA}_{j}^{2}-\frac{2 \sum_{l=1}^{n} u_{i l}^{m} \mathrm{ATA}_{j} \mathrm{ATA}_{l}}{\sum_{k=1}^{n} u_{i k}^{m}}+\frac{\sum_{p=1}^{n} \sum_{q=1}^{n} u_{i p}^{m} u_{i q}^{m} \mathrm{ATA}_{p} \mathrm{ATA}_{q}}{\left(\sum_{k=1}^{n} u_{i k}^{m}\right)^{2}}\right) \\
& +w_{\text {check }} \sum_{i=1}^{c} \sum_{j=1}^{n} u_{i j}^{m} d_{\text {Check }_{i}, \text { Check }_{j}}^{2}
\end{aligned}
$$

$$
\begin{array}{ll}
\text { s.t. } & \sum_{i=1}^{c} u_{i j}=1, \quad \forall j=1, \ldots, n \\
& u_{i j} \geq 0, \quad \forall i=1, \ldots, c, j=1, \ldots, n \\
& \mathrm{PH}_{i}<\mathrm{PH}_{\max }, \quad \forall i=1, \ldots, c \\
& \widetilde{P}_{i}>\underset{\sim}{P}, \quad \forall i=1, \ldots, c .
\end{array}
$$


TABLE 9: Similarities of checks.

\begin{tabular}{lccccc}
\hline & LU/SV & OP/VC & IN/FC & RS & DS \\
\hline LU/SV & 1 & 0.5 & 0.5 & 0.2 & 0.2 \\
OP/VC & 0.5 & 1 & 0.6 & 0.25 & 0.25 \\
IN/FC & 0.5 & 0.6 & 1 & 0.3 & 0.3 \\
RS & 0.2 & 0.25 & 0.3 & 1 & 0.6 \\
DS & 0.2 & 0.25 & 0.3 & 0.6 & 1 \\
\hline
\end{tabular}

4.1.2. The Flow of Optimal Combination of Maintenance Tasks. Based on the clustering model, Model (31) is supplemented with two additional constraints. Besides, the constraints are difficult to be explicitly represented by $u_{i j}, c_{i}$. It is not suitable to directly solve the maintenance tasks combination problem as a normal clustering problem. However, maintenance tasks can be clustered first, and then the result of clustering should be examined or checked whether clustering results satisfy constraints. If clustering results do not meet the requirements, we increase the number of clustering and recomputation until the results satisfy the constraint [20]. Its detailed steps are described in Algorithm 7.

\section{Algorithm 7.}

Step 1. Initialize the parameter and set $c=2$.

Step 2. Solve clustering model (31).

Step 2.1. Set $k=1, \kappa=1.5, \sigma_{1}=500$.

Step 2.2. By the penalty function, the clustering model is transformed into a unconstrained optimization problem as $y(U)=J_{m}(U)+\sigma_{k} P(U)$, where $P(U)=$ $\left(\sum_{i=1}^{c} \sum_{j=1}^{n} \max ^{2}\left(0,-u_{i j}\right)+\sum_{j=1}^{n}\left(\sum_{i=1}^{c} u_{i j}-1\right)^{2}\right), J_{m}(U)$ is (31).

Step 2.3. Optimize the unconstrained optimization problem $\min y(U)$ by Algorithms 3 or 4 or 5 .

Step 2.4. When $\sigma_{k} P(U)<\varepsilon$, go to Step 3. Otherwise, set $\sigma_{k+1}=\kappa \sigma_{k}, k=k+1$ and go to Step 2.3.

Step 3. Tasks in the same class are taken as same maintenance work package. The man-hours $\mathrm{PH}_{i}$ of the $i$ th maintenance work package Package ${ }_{i}$ is totaled by (28) or (29). Summarize the maintenance interval $P P_{i}=\left[\underset{\sim i}{P}, \widetilde{P}_{i}\right]$ by $(30)$.

Step 4. Determine whether $\mathrm{PH}_{i}<\mathrm{PH}_{\max }, \widetilde{P}_{i}>\underset{\sim}{P}, \forall i=$ $1, \ldots, c$ are satisfied. When $\mathrm{PH}_{i}<\mathrm{PH}_{\max }, \widetilde{P}_{i}>\underset{\sim}{P}, \forall i=$ $1, \ldots, c$, it indicates that the current clustering result is a reasonable maintenance task combination. Otherwise, $\operatorname{set} c=$ $c+1$, go to Step 2 and compute again.

Step 5. Analyze and compare maintenance cost between before and after the combination.

Step 2 to Step 4 form an inner loop, in which the preset number of classes is increased constantly until a combination of maintenance task satisfies the constraints.
4.2. Instances of Combination Optimization of Aircraft Maintenance Tasks. Some scheduled maintenance tasks of certain aircraft are investigated from an airline company, shown in Table 10, where the maintenance interval is measured by flight hours. Judging from engineering experience, we set $w_{\text {period }}=0.8, w_{\text {zone }}=0.05, w_{\text {check }}=0.1$, and $w_{\text {ATA }}=0.05$. $\mathrm{PH}_{i}$ is computed by (28), and set $\mathrm{PH}_{\max }=2$. Algorithm 7 is employed to find out optimal combination of scheduled maintenance tasks in Table 10 in order to form maintenance work package. When penalty function is utilized to solve unconstraint clustering problems, set $\kappa=1.5$ and $\sigma_{1}=500$. Algorithm 5 is applied to solve unconstraint optimization problem, where $\mu_{\text {outer }}, \mu_{\text {inner }}$ are computed by (9) and $\alpha$ is determined with (16). Let other parameters as the following $\mu_{\text {inner }}=0.5, \mu_{\text {outer }}=1.4, \mu^{e}=1.2, \mu^{o c}=0.7, \mu^{i c}=1.5, \mu^{s}=$ $0.3, \dot{\lambda}_{h}=2, \ddot{\lambda}_{h}=0.9, \dddot{\lambda}_{h}=0.985, t_{\max }=10, \rho_{\text {nei }}=0.1$, $h_{\text {small }}=0.1, \alpha^{\text {forward }}=2, \alpha^{\text {reverse }}=-2, \alpha^{f e}=2, \alpha^{f c}=0.65$, $\alpha^{f s}=-1$, and $\alpha^{r e}=2, \alpha^{r c}=0.6$. The result of Algorithm 7 is tabulated in Table 11, from which it can be known that seventeen maintenance tasks are combined into thirteen maintenance work packages.

Suppose costs of every maintenance includes four parts: labor costs, device costs, halt costs, and fixed costs, which are, respectively, represented as Cost ${ }_{l}$, Cost $_{d}$, Cost ${ }_{h}$, and Cost $f$. Specifically, the fixed cost typically refers to the depreciation of maintenance tools and fixed assets and the management fees. Cost $h$ and Cost $f$ are always the same for any repair. Cost $_{d}$, which is determined by maintenance items, is the total device costs of all maintenance items repaired and is irrelative with the way of combination. Thereby, maintenance cost before the combination is Cost $_{b}=F_{\text {labour }} \sum_{j=1}^{17}$ Hour $_{j}+$ $\sum_{j=1}^{17}$ Device $_{j}+17$ Cost $_{h}+17$ Cost $_{f} . F_{\text {labour }}$ refers to the labor costs per unit time. Device ${ }_{j}$ indicates the device cost of the $j$ th maintenance item. After the combination, the maintenance cost will be Cost $t_{a}=F_{\text {labour }} \sum_{j=1}^{13} \mathrm{PH}_{j}+\sum_{j=1}^{17}$ Device $_{j}+$ $13 \operatorname{Cost}_{h}+$ 13Cost $_{f}$. The maintenance cost is compared between before and after combination is tabulated in Table 12 . As $\operatorname{Cost}_{b}-\operatorname{Cost}_{a}=4 \operatorname{Cost}_{h}+4 \operatorname{Cost}_{f}$, it can be concluded that the combination helps saving cost and improving efficiency. Halt cost will cause great cost, which accounts for a large proportion in the total maintenance cost. Therefore, optimal combination of maintenance tasks can greatly increase profits of the airlines.

\section{Conclusions and Discussions}

Firstly, the improved fuzzy C-means clustering model is presented, which can be directly solved. Considering FCM model has many stationary points, the optimization method which needs derivatives is not applicable to solve it. Based on the positive bases theory, center ray operation is introduced and a novel nonlinear simplex optimization method is developed. Besides, its convergences are roughly analyzed. As a direct optimization method, it can be utilized to solve the clustering model effectively. Finally, the improved FCM model and the novel optimization method are employed to optimally combine maintenance tasks and exhibit good effects. 
TABLE 10: Some scheduled maintenance tasks of an airline.

\begin{tabular}{|c|c|c|c|c|c|c|}
\hline \multirow{2}{*}{ Number } & \multirow{2}{*}{ ATA } & \multicolumn{2}{|c|}{ Maintenance interval } & \multirow{2}{*}{ Check } & \multirow{2}{*}{ Zone } & \multirow{2}{*}{ Man-hours } \\
\hline & & Lower boundary & Upper boundary & & & \\
\hline 1 & 2410 & 338 & 394 & IN/FC & 210 & 0.1 \\
\hline 2 & 3201 & 240 & 278 & RS & $734 ; 744$ & 0.05 \\
\hline 3 & 3201 & 221 & 258 & RS & $734 ; 744$ & 0.05 \\
\hline 4 & 5381 & 1543 & 1779 & $\mathrm{IN} / \mathrm{FC}$ & $121 ; 122$ & 0.3 \\
\hline 5 & 5780 & 2746 & 3176 & IN/FC & $510 ; 520 ; 530 ; 540 ; 550 ; 560 ; 570$ & 0.3 \\
\hline 6 & 3243 & 1231 & 1468 & IN/FC & 310 & 0.05 \\
\hline 7 & 3301 & 1228 & 1417 & $\mathrm{OP} / \mathrm{VC}$ & 200 & 0.02 \\
\hline 8 & 5280 & 2359 & 2760 & IN/FC & $820 ; 830 ; 840$ & 0.4 \\
\hline 9 & 2304 & 895 & 1014 & $\mathrm{OP} / \mathrm{VC}$ & 211 & 0.1 \\
\hline 10 & 2702 & 12331 & 14161 & LU/SV & $133 ; 570$ & 0.5 \\
\hline 11 & 2702 & 7920 & 9242 & LU/SV & $134 ; 672$ & 0.4 \\
\hline 12 & 2703 & 16116 & 18200 & $\mathrm{IN} / \mathrm{FC}$ & $210 ; 572 ; 672$ & 0.4 \\
\hline 13 & 2704 & 23413 & 27600 & $\mathrm{IN} / \mathrm{FC}$ & $133 ; 134 ; 325$ & 0.4 \\
\hline 14 & 2709 & 9788 & 11258 & IN/FC & $333 ; 334 ; 343 ; 344$ & 1 \\
\hline 15 & 2704 & 28497 & 32543 & IN/FC & $131 ; 134 ; 325$ & 0.7 \\
\hline 16 & 2704 & 24885 & 29716 & $\mathrm{IN} / \mathrm{FC}$ & $131 ; 134 ; 325$ & 0.4 \\
\hline 17 & 2713 & 11671 & 13050 & DS & $133 ; 134 ; 211 ; 541 ; 542 ; 543 ; 544 ; 553$ & 0.5 \\
\hline
\end{tabular}

TABLE 11: Optimal work package after the combination of maintenance tasks.

\begin{tabular}{|c|c|c|c|c|}
\hline \multirow{2}{*}{ Number } & \multicolumn{2}{|c|}{ Maintenance interval } & \multirow{2}{*}{ Man-hours of package } & \multirow{2}{*}{ Tasks } \\
\hline & Lower boundary & Upper boundary & & \\
\hline 1 & 338 & 394 & 0.1 & 1 \\
\hline 2 & 240 & 258 & 0.1 & 2,3 \\
\hline 3 & 1543 & 1779 & 0.3 & 4 \\
\hline 4 & 2746 & 2760 & 0.7 & 5,8 \\
\hline 5 & 1231 & 1417 & 0.07 & 6,7 \\
\hline 6 & 895 & 1014 & 0.1 & 9 \\
\hline 7 & 12331 & 14161 & 0.5 & 10 \\
\hline 8 & 7920 & 9242 & 0.4 & 11 \\
\hline 9 & 16116 & 18200 & 0.4 & 12 \\
\hline 10 & 23413 & 27600 & 0.4 & 13 \\
\hline 11 & 9788 & 11258 & 1 & 14 \\
\hline 12 & 28497 & 29716 & 1.1 & 15,16 \\
\hline 13 & 11671 & 13050 & 0.5 & 17 \\
\hline
\end{tabular}

TABLE 12: Optimal work package after the combination of maintenance tasks.

\begin{tabular}{lccc}
\hline & Before combination & After combination & Cost saved \\
\hline Labor cost & $F_{\text {labour }} \sum_{j=1}^{17}$ Hour $_{j}$ & $F_{\text {labour }} \sum_{j=1}^{13} \mathrm{PH}_{j}$ & 0 \\
Device cost & $\sum_{j=1}^{17}$ Device $_{j}$ & $\sum_{j=1}^{17}$ Device $_{j}$ & 0 \\
Halt cost & 17 Cost $_{h}$ & 13 Cost $_{h}$ & 4 Cost $_{h}$ \\
Fixed cost & 17 Cost $_{f}$ & $13 \operatorname{Cost}_{f}$ & 4 Cost $_{f}$ \\
\hline
\end{tabular}

However, there are still several problems to be further discussed. (1) The number of independent variables contained in the improved clustering model is still large. In case of a large number of samples, it is still inconvenient to solve FCM directly; while the clustering model, which is easier to be solved, needs to be further studied. (2) The simplex algorithm proposed in the paper is much more effective than the traditional algorithm. However, sometimes it is likely that the improved method fails to achieve good result because the searching direction can not be adjusted timely. From this view, the algorithm has to be improved. The most prominent point is that a certain rotation operation should be taken in the simplex algorithm to change the search directions. (3) $h_{m}$ has great influences on the performance of the improved algorithm proposed in the paper. Although it is proved to be feasible that $h_{m}$ is taken as a decreasing exponential function, the efficiency is still to be improved. Various factors can be associated to find out other way to control changes of $h_{m}$ so as to improve the performance. (4) The experimental results show that the algorithm proposed in the paper can 
rapidly approach to the nearby region of the extreme point from the far initial point. However, it takes a long time to converge to an accurate solution within the neighbourhood of the extreme point. For this reason, how to improve local convergence performance of the algorithm should be an important research issue. (5) For the optimal combination of maintenance tasks, maintenance tasks are simplified and only partial properties are selected to measure similarities. Although it is proved to be practical in most engineering problems, the result is acceptable but not optimal due to the ignorance of details of the execution of maintenance tasks such as maintenance tools and maintenance orders. A more economical combination will be achieved if complete and comprehensive factors can be taken into consideration to determine similarities of maintenance tasks.

\section{Conflict of Interests}

The authors declare that there is no conflict of interests regarding the publication of this paper.

\section{Acknowledgments}

The paper is supported by funds including the National Natural Science Foundation of China (no. 61403198, no. 61079013, and no. 61079014), Youth Foundation of Jiangsu Province in China (no. BK20140827), Key Programs of Natural Science Foundation of China and China Civil Aviation Joint Fund (no. 60939003), and Natural Science Foundation of Jiangsu Province in China (no. BK2011737).

\section{References}

[1] C. Luo and B. Yu, "Low dimensional simplex evolution: a new heuristic for global optimization," Journal of Global Optimization, vol. 52, no. 1, pp. 45-55, 2012.

[2] K.-H. Chang, "Stochastic Nelder-Mead simplex method-a new globally convergent direct search method for simulation optimization," European Journal of Operational Research, vol. 220, no. 3, pp. 684-694, 2012.

[3] R. Michael, R. M. Lewis, and V. Torczon, Rank ordering and positive basis in pattern search algorithms, Daa, 1998.

[4] K. I. M. Mckinnon, "Convergence of the Nelder-Mead simplex method to a nonstationary point," Society for Industrial and Applied Mathematics, vol. 9, no. 1, pp. 148-158, 1998.

[5] J. C. Lagarias, J. A. Reeds, M. H. Wright, and P. E. Wright, "Convergence properties of the Nelder-Mead simplex method in low dimensions," SIAM Journal on Optimization, vol. 9, no. 1, pp. 112-147, 1998.

[6] L. Han and M. Neumann, "Effect of dimensionality on the Nelder-Mead simplex method," Optimization Methods \& Software, vol. 21, no. 1, pp. 1-16, 2006.

[7] Q. Wang and S. Ju, Research of Novel Fuzzy Clustering Algorithms Based on Objective Function and Its Applications, Jiangsu University, 2014.

[8] I. Berget, B.-H. Mevikc, and T. Næs, "Novel modifications and applications of fuzzy C-means methodology," Computational Statistics and Data Analysis, vol. 52, pp. 2403-2418, 2008.
[9] V. Torczon, Multi-directional search: a direct search algorithm for parallel machines [Ph.D. thesis], Rice University, Houston, Tex, USA, 1989.

[10] C. J. Price, I. D. Coope, and D. Byatt, "A convergent variant of the Nelder-Mead algorithm," Journal of Optimization Theory and Applications, vol. 113, no. 1, pp. 5-19, 2002.

[11] I. D. Coope and C. J. Price, "Frame based methods for unconstrained optimization," Journal of Optimization Theory and Applications, vol. 107, no. 2, pp. 261-274, 2000.

[12] T. Liao and T. Stutzle, "Benchmark results for a simple hybrid algorithm on the CEC 2013 benchmark set for real-parameter optimization," in Proceedings of the IEEE Congress on Evolutionary Computation (CEC '13), pp. 1938-1944, Cancun, Mexico, June 2013.

[13] C. J. Price and I. D. Coope, "Frame-based ray search algorithms in unconstrained optimization," Journal of Optimization Theory and Applications, vol. 116, no. 2, pp. 359-377, 2003.

[14] A. K. Muchiri, Maintenance Planning Optimisation for the Boeing 737 Next Generation, Delft University of Technology, 2002.

[15] G. Cabanes, Y. Bennani, R. Destenay, and A. Hardy, "A new topological clustering algorithm for interval data," Pattern Recognition, vol. 46, no. 11, pp. 3030-3039, 2013.

[16] C. W. D. de Almeida, R. M. C. R. de Souza, and A. L. B. Candeias, "Fuzzy Kohonen clustering networks for interval data," Neurocomputing, vol. 99, pp. 65-75, 2013.

[17] B. A. Pimentel and R. M. C. R. de Souza, "A weighted multivariate Fuzzy C-Means method in interval-valued scientific production data," Expert Systems with Applications, vol. 41, no. 7, pp. 3223-3236, 2014.

[18] C.-H. Kao, J. Nakano, S.-H. Shieh et al., "Exploratory data analysis of interval-valued symbolic data with matrix visualization," Computational Statistics \& Data Analysis, vol. 79, pp. 14-29, 2014.

[19] A. B. Dos S. Dantas and F. de A. T. De Carvalho, "Adaptive batch SOM for multiple dissimilarity data tables," in Proceedings of the 23rd IEEE International Conference on Tools with Artificial Intelligence (ICTAI '11), pp. 575-578, IEEE, Boca Raton, Fla, USA, November 2011.

[20] J. F. Kolen and T. Hutcheson, "Reducing the time complexity of the fuzzy c-means algorithm," IEEE Transactions on Fuzzy Systems, vol. 10, no. 2, pp. 263-267, 2002. 


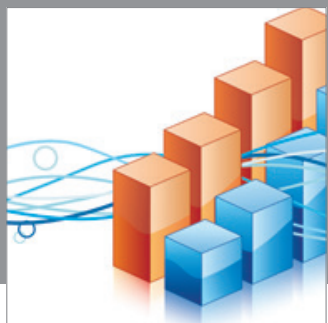

Advances in

Operations Research

mansans

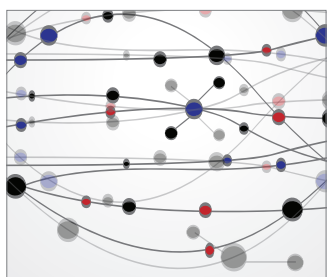

The Scientific World Journal
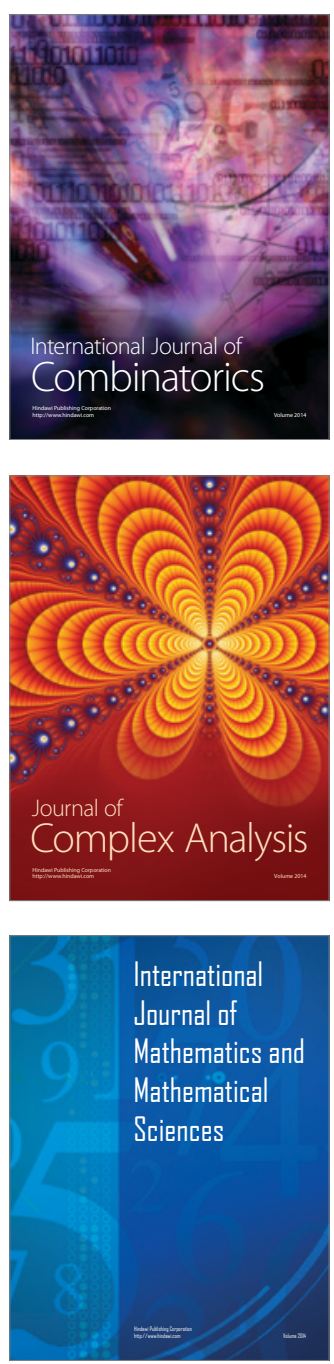
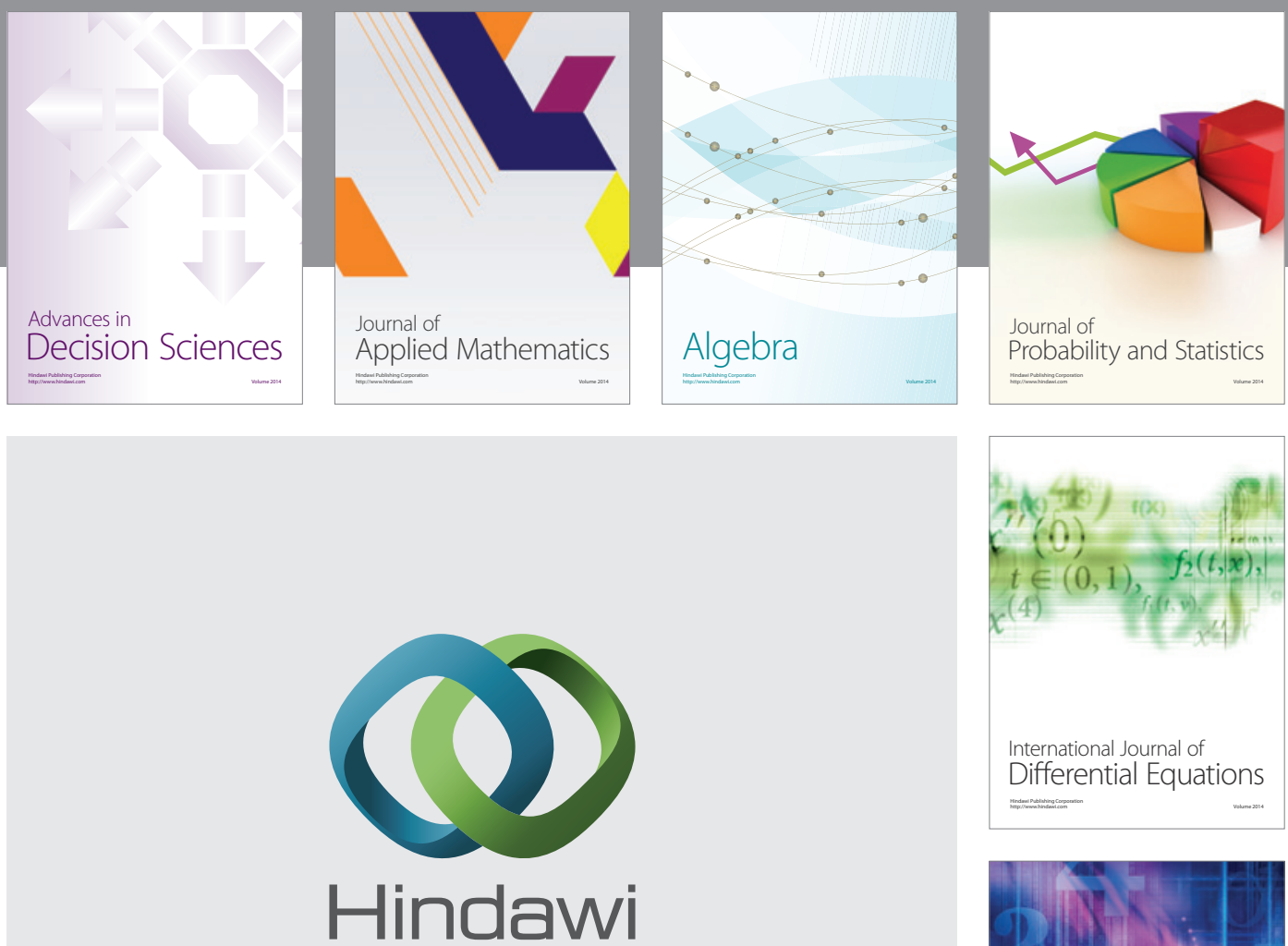

Submit your manuscripts at http://www.hindawi.com
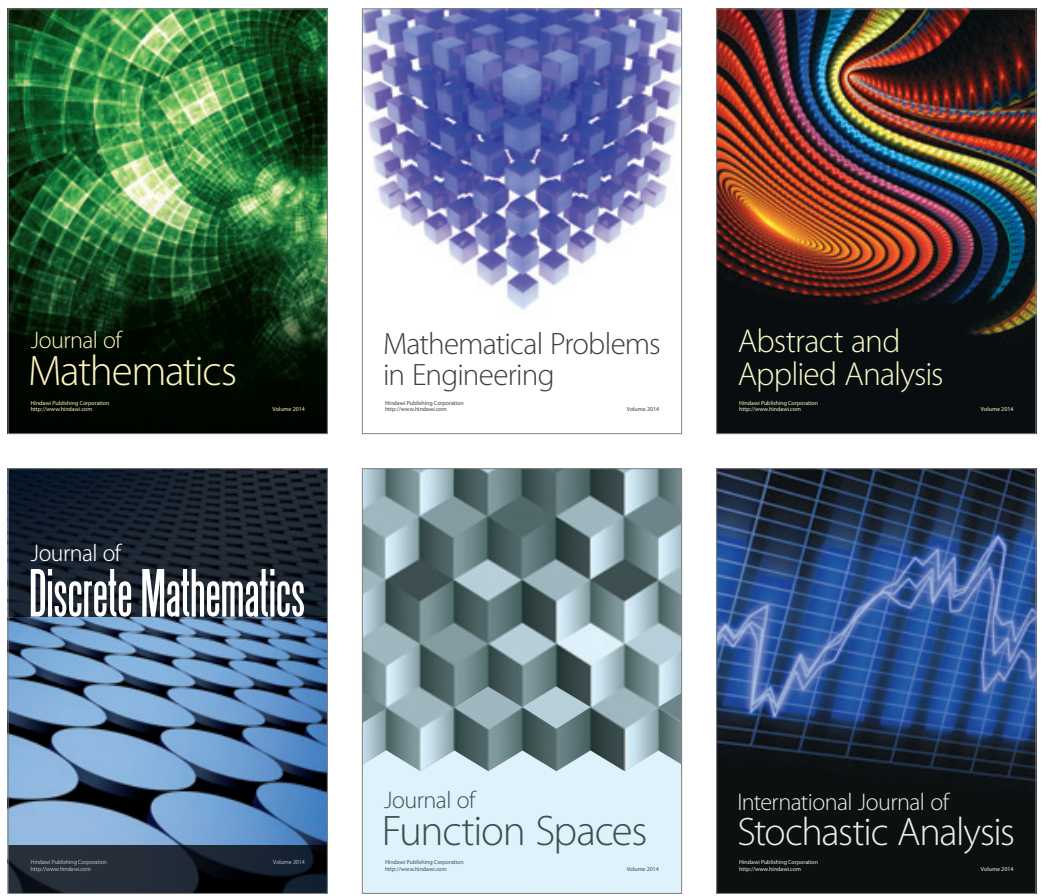

Journal of

Function Spaces

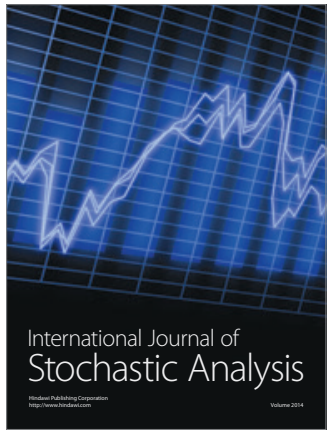

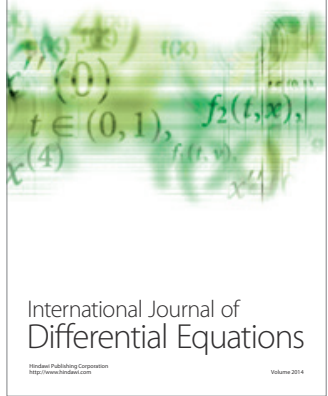
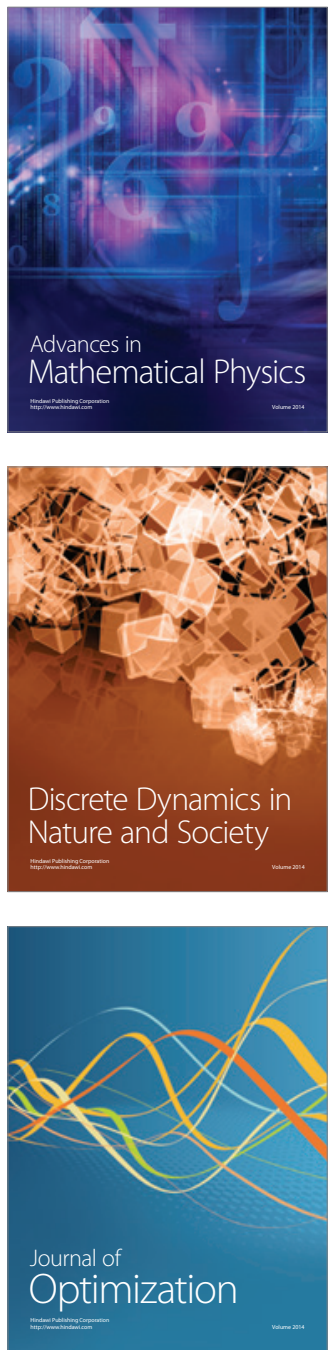